\title{
Dynamic Analysis of Beddington-DeAngelis Predator-Prey System with Nonlinear Impulse Feedback Control
}

\author{
Dezhao Li, ${ }^{1}$ Huidong Cheng $\mathbb{D}^{1},{ }^{1}$ and Yu Liu ${ }^{2}$ \\ ${ }^{1}$ College of Mathematics and System Sciences, Shandong University of Science and Technology, \\ Qingdao 266590, Shandong, China \\ ${ }^{2}$ College of Foreign Languages, Shandong University of Science and Technology, \\ Qingdao 266590, Shandong, China
}

Correspondence should be addressed to Huidong Cheng; chd900517@sdust.edu.cn

Received 4 September 2019; Revised 15 October 2019; Accepted 28 October 2019; Published 22 November 2019

Guest Editor: Alejandro Hossian

Copyright (@ 2019 Dezhao Li et al. This is an open access article distributed under the Creative Commons Attribution License, which permits unrestricted use, distribution, and reproduction in any medium, provided the original work is properly cited.

\begin{abstract}
In this paper, a predator-prey system with pesticide dose-responded nonlinear pulse of Beddington-DeAngelis functional response is established. First, we construct the Poincare map of the impulsive semidynamic system and discuss its main properties including the monotonicity, differentiability, fixed point, and asymptote. Second, we address the existence and globally asymptotic stability of the order-1 periodic solution and the sufficient conditions for the existence of the order- $k(k \geq 2)$ periodic solution. Thirdly, we give the threshold conditions for the existence and stability of boundary periodic solutions and present the parameter analysis. The results show that the pesticide dosage increases with the extension of the control period and decreases with the increase of the threshold. Besides, the state pulse feedback control can manage the pest population at a certain level and avoid excessive application of pesticides.
\end{abstract}

\section{Introduction}

The predator-prey system plays an important role in the relationship of biological populations, so many predatorprey systems with different functional responses have been studied, such as the Monod type [1-5], the Holling type [6-13], and the Ivlev type [14-18]. Currently, the use of chemicals is more and more widespread in agriculture. Also, when the number of pests reaches a critical value, we can release natural enemies. Therefore, the feedback control of pulse state is proposed [17, 19-22]. The BeddingtonDeAngelis functional response was introduced by Beddington [23] and DeAngelis et al. [24]. The BeddingtonDeAngelis functional response avoided some of the singular behaviors of the ratio-dependent model at low density [25]. Cantrell and Cosner discussed the following predator-prey system with Beddington-DeAngelis functional response $[23,24,26]$ :

$$
\left\{\begin{array}{l}
u^{\prime}(t)=r u\left(1-\frac{u}{K}\right)-\frac{m u v}{(a+b v+c u)} \\
v^{\prime}(t)=v\left(\frac{\varepsilon m u}{(a+b v+c u)}-\mu\right), \\
u(0)=u_{0}>0 \\
v(0)=v_{0}>0
\end{array}\right.
$$

where $r, K, m, a, b, c, \varepsilon$, and $\mu$ are positive constants. $u(t)$ and $v(t)$ represent the population density of prey and predator at time $t, K$ is the environmental carrying capacity of the prey, and $r$ is the intrinsic growth rate of prey. Function $m u /(a+b v+c u)$ indicates the Beddington-DeAngelis functional response, and $b v$ stands for mutual interference between the predators. The constants $\varepsilon$ and $\mu$ represent the rate of conversion and death rate of predators, respectively. 
For simplicity, we determine dimensionless system (1) and scale it as follows:

$$
\begin{aligned}
& t \longrightarrow r t, \\
& u \longrightarrow \frac{u}{K}, \\
& v \longrightarrow \frac{b v}{c K} .
\end{aligned}
$$

Then, we get

$$
\left\{\begin{array}{l}
u^{\prime}(t)=u(1-u)-\frac{s u v}{(u+v+A)}, \\
v^{\prime}(t)=\delta v\left(\frac{u}{(u+v+A)}-d\right), \\
u(0)=u_{0}>0, \\
v(0)=v_{0}>0,
\end{array}\right.
$$

where

$$
\begin{aligned}
& s=\frac{m}{b r}, \\
& \delta=\frac{m \varepsilon}{c r}, \\
& d=\frac{c \mu}{m \varepsilon}, \\
& A=\frac{a}{c K} .
\end{aligned}
$$

In recent years, many pulse equations have been studied that simulate the ecological processes of populations, and most of these studies are pulse differential equations at the fixed time [1, 27-32]. However, feedback control of time pulse has certain defects, which may reduce crop yield and possibly increase management costs. Therefore, we can choose to spray the pesticide when the quantity of pests reaches a certain threshold instead of spraying the pesticide at a fixed time. This measure avoids the possibility of the explosive growth of the number of pests and is more suitable for pest control. This paper studies the BeddingtonDeAngelis system with pulse state feedback control strategies:

$$
\left\{\begin{array}{l}
u^{\prime}(t)=u(1-u)-\frac{s u v}{(u+v+A)} \\
v^{\prime}(t)=\delta v\left(\frac{u}{(u+v+A)}-d\right) \\
u\left(t^{+}\right)=P(D) u(t) \\
v\left(t^{+}\right)=Q(D) v(t)+\tau
\end{array}\right\} u \neq \mathrm{TH},
$$

Capturing or using chemicals on predators and prey may impulsively reduce the density of the predators and prey. $P(D)$ and $Q(D)$ represent the survival rate of prey and predator populations when a given dose $D$ of insecticides is applied and $0 \leq P(D)<1$ and $0 \leq Q(D)<1$. We assume that insecticides have different insecticidal rates for these two populations, where $P(D)=e^{-k_{1} D}$ and $Q(D)=e^{-k_{2} D}, \tau \geq 0$ is the constant number of natural enemies released $[33,34]$.

Some published articles focus on the property of the successor function and Poincaré map to discuss the existence of order-1 periodic solution and the local stability. Besides, if the proposed model has the first integrals, the existence of order-2 periodic solutions can be discussed [35-40]. However, due to the complexity of such model as the Beddington-DeAngelis system in this paper, the problem of global dynamics such as the global stability of the model and the existence of order- $k(k \geq 2)$ periodic solution has not been well solved. Also, there a few researches on the property of Poincaré map while it is applied. So the main arrangement of this paper is as follows. In Section 2, some preliminaries about the pulse semidynamic system and system (3) are given. In Section 3, we construct the Poincaré map, deduce the expression of Poincare map function of system (5), and then give some of its properties such as the monotonicity, differentiability, fixed point, and asymptote. In Section 4, we prove the existence and stability of the boundary periodic solution and order- $k(k \geq 1)$ periodic solution of system (5). In Section 5, we conducted a numerical simulation.

\section{Preliminaries}

2.1. Preliminaries of Pulse Semidynamic Systems. A pulsed semipowered system with state-dependent feedback control can be expressed as [41, 42]

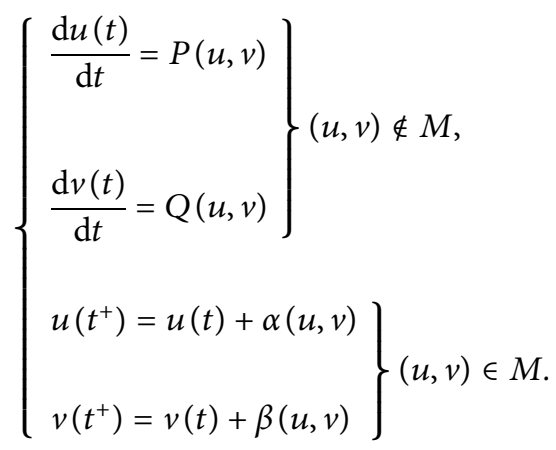

Here, $(u, v) \in R^{2}, P, Q, \alpha$, and $\beta$ are continuous functions from $R^{2}$ to $R$. Let $M \subset R^{2}$ be the impulse set of system (6), and for any $f(u, v) \in M$, the impulse occurs; the map $I$ is defined as

$$
f^{+}=I(f)=(u+\alpha(u, v), v+\beta(u, v))=\left(u^{+}, v^{+}\right) \in R^{2},
$$

where $f^{+}$is the impulse point for $f$. We define $N=I(M)$ as the phase set of the system and $N \cap M=\varnothing, X=R^{2}$ is the metric space, and $R_{+}$is the set of all nonnegative reals; we call $(X, \Pi, R)$ as a semidynamic system. For any $f \in X$, 
$\Pi(f, 0)=f, \Pi(\Pi(f, t), s)=\Pi(f, t+s)$, where $t, s \in R_{+}$ [43]. The set

$$
D^{+}(f)=\left\{\Pi(f, t) \mid t \in R_{+}\right\},
$$

is called the positive orbit of $f$. Furthermore, for any set $M \in X$, let

$$
M^{+}(f)=D^{+}(f) \cap M-\{f\} .
$$

Next, we give the definition of impulsive semidynamic system and order- $k$ periodic solution.

Definition 1 (see [44, 45]). The pulsed semidynamic system $(X, \Pi, M, I)$ consists of the nonempty closed subset $M$ of $X$, the continuous semidynamic system $(X, \Pi)$, and the continuous function $I$.

We denote the points of discontinuity of $\Pi_{f}$ by $\left\{f_{n}^{+}\right\}$and call $f_{n}^{+}$an impulsive point of $f_{n}$. We define a function $\Phi$ from $X$ into the extended positive reals $R_{+} \cup\{\infty\}$ as follows: let $f \in X$; if $M^{+}(f)=\varnothing$, we set $\Phi(f)=\infty$; otherwise $M^{+}(f) \neq \varnothing$, and we set $\Phi(f)=s$, where $\Pi(f, t) \notin M$ for $0<t<s$ but $\Pi(f, t) \in M$.

Definition 2 (see [46]). For trajectory $\Pi_{f}$ in $(X, \Pi, M, I)$, if there are nonnegative integers $m \geq 0$ and $k \geq 1, k$ is the minimum integer satisfying $f_{m}^{+}=f_{m+k}^{+}$and $T_{k}=\sum_{i=m}^{m+k-1}$
$\Phi\left(f_{i}\right)=\sum_{i=m}^{m+k-1} s_{i}$; then, the period of $\Pi_{f}$ is $T$, and there is a period of order- $k$.

Definition 3 (see $[42,47])$. The T-periodic solution $(u, v)=$ $(\xi(t), \eta(t))$ of thwe system

$$
\left\{\begin{array}{l}
\frac{\mathrm{d} u}{\mathrm{~d} t}=P(u, v), \\
\frac{\mathrm{d} v}{\mathrm{~d} t}=Q(u, v), \quad \text { if } \phi(u, v) \neq 0, \\
u^{+}=u+\alpha(u, v), \\
v^{+}=v+\beta(u, v), \quad \text { if } \phi(u, v)=0,
\end{array}\right.
$$

is orbitally asymptotically stable and enjoys the property of asymptotic phase if $\mu_{1}$ satisfies $\left|\mu_{1}\right|<1$, where

$$
\mu_{1}=\prod_{k=1}^{q} \Delta_{k} \exp \left(\int_{0}^{T}\left[\frac{\partial P}{\partial u}(\xi(t), \eta(t))+\frac{\partial Q}{\partial v}(\xi(t), \eta(t))\right] \mathrm{d} t\right)
$$

with

$$
\Delta_{k}=\frac{P_{+}((\partial \beta / \partial v)(\partial \phi / \partial u)-(\partial \beta / \partial u)(\partial \phi / \partial v)+(\partial \phi / \partial u))+Q_{+}((\partial \alpha / \partial u)(\partial \phi / \partial v)-(\partial \alpha / \partial v)(\partial \phi / \partial u)+(\partial \phi / \partial u))}{P(\partial \phi / \partial u)+Q(\partial \phi / \partial v)}
$$

and $\phi$ is continuously differentiable with respect to $u$ and $v$. $(u, v) \notin M$ can also be denoted by $\phi(u, v) \neq 0 . P, Q, \partial \alpha / \partial u$, $\partial \alpha / \partial v, \partial \beta / \partial u, \partial \beta / \partial v, \partial \phi / \partial u$, and $\partial \phi / \partial v$ are calculated at the point $\quad\left(\xi\left(t_{k}\right), \eta\left(t_{k}\right)\right), \quad P_{+}=P\left(\xi\left(t_{k}^{+}\right), \eta\left(t_{k}^{+}\right)\right), \quad$ and $Q_{+}=Q\left(\xi\left(t_{k}^{+}\right), \eta\left(t_{k}^{+}\right)\right)$.

2.2. Preliminaries of System (3). We know that the solution of system (3) is positive and bounded for all $t$. If $d \geq(1+A)^{-1}$, then the equilibrium point $(1,0)$ is globally asymptotically stable [25].

If $0<d<(1+A)^{-1}$, system (3) has three equilibrium points, $(0,0),(1,0)$, and $\left(u_{*}, v_{*}\right)$, respectively, where $u_{*}$ and $v_{*}$ are all positive and satisfy the following conditions:

$$
\left\{\begin{array}{l}
1-u_{*}-\frac{s v_{*}}{u_{*}+v_{*}+A}=0, \\
\frac{u_{*}}{u_{*}+v_{*}+A}=d .
\end{array}\right.
$$

For completeness, we summarize global results for system (3) in Lemma $1[25,48,49]$.

\section{Lemma 1}

(i) If $d>(1+A)^{-1},(1,0)$ is globally asymptotically stable (ii) If $d<(1+A)^{-1}$ and $\operatorname{tr}\left(J\left(u_{*}, v_{*}\right)\right) \leq 0$, the $\left(u_{*}, v_{*}\right)$ is globally asymptotically stable

(iii) If $d<(1+A)^{-1}$ and $\operatorname{tr}\left(J\left(u_{*}, v_{*}\right)\right)>0$, there is an exact limit cycle

\section{Poincaré Map}

3.1. Domains of the Poincaré Map. In the following parts, we only discuss the case of $\left(u_{*}, v_{*}\right)$ as the globally asymptotically stable point of system (5).

System (5) has two isoclinal lines, which are defined as $L_{1}$ and $L_{2}$ :

$$
\begin{aligned}
& L_{1}: v=\frac{(1-A) u-u^{2}+A}{s+u-1} ; \\
& L_{2}: v=\frac{(1-d) u}{d}-A .
\end{aligned}
$$

Next, the lines associated with the phase set and impulse set are defined as $L_{3}$ and $L_{4}$ :

$$
\begin{aligned}
& L_{3}: u=e^{-k_{1} D_{\mathrm{TH}}} \\
& L_{4}: u=\mathrm{TH} .
\end{aligned}
$$

In this case, the value range of $\mathrm{TH}$ is $0<\mathrm{TH}<u_{*}$ and lines $L_{3}$ and $L_{4}$ always intersect with line $L_{1}$. The intersection 
point of $L_{1}$ and $L_{4}$ presents as $G\left(\mathrm{TH}, v_{G}\right)$, and the intersection point of $L_{1}$ and $L_{3}$ presents as $H\left(e^{-k_{1} D} \mathrm{TH}, v_{H}\right)$, where $v_{G}$ and $v_{H}$ are, respectively,

$$
\begin{aligned}
& v_{G}=\frac{(1-A) \mathrm{TH}-\mathrm{TH}^{2}+A}{\mathrm{TH}+s-1}, \\
& v_{H}=\frac{(1-A) e^{-k_{1} D} \mathrm{TH}-e^{k_{1}^{2} D^{2}} \mathrm{TH}^{2}+A}{e^{-k_{1} D} \mathrm{TH}+s-1} .
\end{aligned}
$$

The open set defined in $R_{+}^{2}$ is as follows:

$$
\Omega=\{(u, v) \mid u>0, v>0, u<\mathrm{TH}\} \subset R_{+}^{2} .
$$

The impulse set $M$ is the part of line $L_{4}$ above the $U$-axis and below point $G$ :

$$
M=\left\{(u, v) \in R^{2} \mid u=\mathrm{TH}, 0 \leq v<\frac{(1-A) \mathrm{TH}-\mathrm{TH}^{2}+A}{\mathrm{TH}+s-1}\right\} .
$$

The continuous function $I$ is expressed as

$$
I:(\mathrm{TH}, v) \in M \longrightarrow\left(u^{+}, v^{+}\right)=\left(e^{-k_{1} D} \mathrm{TH}, e^{-k_{2} D} v+\tau\right) \in R^{2}
$$

so the phase set $N$ is

$$
N=I(M)=\left\{\left(u^{+}, v^{+}\right) \mid u^{+}=e^{-k_{1} D_{1}} \mathrm{TH}, v^{+} \in \sigma\right\},
$$

where $\sigma=\left[\tau, e^{-k_{2} D}\left(\left((1-A) \mathrm{TH}-\mathrm{TH}^{2}+A\right) /(\mathrm{TH}+s-1)\right)+\right.$ $\tau$ ]. We assume that the initial point $\left(u_{0}^{+}, v_{0}^{+}\right)$is always on the $L_{3}$ in the following sections.

3.2. Construction of Poincaré Map. The Poincaré map of system (5) can be defined in different ways. In this work, we choose the $L_{3}$ to define the Poincaré map.

Because $\left(u_{*}, v_{*}\right)$ is chosen as the globally asymptotically stable point in system (5), and the value range of $\mathrm{TH}$ is defined in the impulse set $L_{4}: u=\mathrm{TH}$ as $0<\mathrm{TH}<u_{*}$, any trajectory starting from the point $Z_{k}^{+}\left(e^{-k_{1} D} \mathrm{TH}, v_{k}^{+}\right)$on the phase set must intersect with the impulse set $L_{4}: u=\mathrm{TH}$ at the point $Z_{k+1}\left(\mathrm{TH}, v_{k+1}\right)$. From Cauchy-Lipschitz theorem, we know the value of $v_{k}^{+}$is only determined by $v_{k+1}$; in order to discuss fluently, we make $v_{k+1}=g\left(v_{k}^{+}\right)$. The point $Z_{k+1}$ on the impulse set $L_{4}$ is mapped to the point $Z_{k+1}^{+}\left(e^{-k_{1} D} \mathrm{TH}, v_{k+1}^{+}\right)$on $L_{3}$ after experiencing an impulse. $v_{k+1}^{+}=e^{-k_{2} D} v_{k+1}+\tau$ is obtained by the impulse function $v\left(t^{+}\right)=e^{-k_{2} D} v(t)+\tau$ of system (5). $Z_{k+1}^{+}$is the initial point of the next impulse function on the phase set.

So we can express the Poincaré map of system (5) as

$$
v_{i+1}^{+}=e^{-k_{2} D} g\left(v_{i}^{+}\right)+\tau=\varphi\left(v_{i}^{+}\right) .
$$

We can determine the Poincaré map from the points on the phase set. Next, we infer the expression of Poincaré map function $\varphi$ and discuss its properties according to the expression of $\varphi$.

According to the following formula of system (5),

$$
\left\{\begin{array}{l}
P(u(t), v(t))=u(1-u)-\frac{s u v}{(u+v+A)}, \\
Q(u(t), v(t))=\delta v\left(\frac{u}{(u+v+A)}-d\right) .
\end{array}\right.
$$

We can rewrite system (5) as a scalar differential equation on the phase set:

$$
\left\{\begin{array}{l}
\frac{\mathrm{d} v}{\mathrm{~d} u}=\frac{\delta v(u /(u+v+A)-d)}{u(1-u)-\operatorname{suv} /(u+v+A)}=\rho(u, v), \\
v\left(e^{-k_{1} D} \mathrm{TH}\right)=v_{0}^{+} .
\end{array}\right.
$$

For model (23), we only focus on the region

$$
\Omega_{1}=\left\{(u, v) ; u>0, v>0, v<\frac{(1-A) u-u^{2}+A}{s+u-1}\right\} \text {. }
$$

The function $\rho(x, y)$ is continuous and differentiable in the region $\Omega_{1}$. Besides, let $u_{0}^{+}=e^{-k_{1} D} \mathrm{TH}$ and $v_{0}^{+}=J$, where $J \in N, J<v_{G}$, and $\left(u_{0}^{+}, v_{0}^{+}\right) \in \Omega_{1}$, so

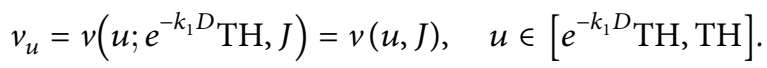

From (23), we get

$$
v(u, J)=J+\int_{e^{-k_{1} D} \mathrm{TH}}^{u} \rho(j, v(j, J)) \mathrm{d} j .
$$

According to (21) and (26), we can obtain the definition of the Poincaré map $\varphi$ :

$$
\varphi(J)=e^{-k_{2} D} v(\mathrm{TH}, J)+\tau .
$$

We simulate a numerical simulation of the Poincaré map function of model (5) (see Figure 1). The two cases are $\varphi\left(v_{H}\right)<v_{H}$ (Figure 1(a)) and $\varphi\left(v_{H}\right)>v_{H}$ (Figure 1(b)).

3.3. The Main Properties of Poincaré Map. Through our analysis of the expression and numerical model of the Poincaré map $\varphi$, and assuming $\tau>0$, the following properties of Poincaré map are given.

Theorem 1. The domain of $\varphi$ is $[0,+\infty)$, and the range of $\varphi$ is $\left[\tau, \varphi\left(v_{H}\right)\right]$, where $\varphi\left(v_{H}\right)=v\left(e^{-k_{1} D} T H, v_{H}\right)+\tau$. $\varphi$ monotonically increases on $\left[0, v_{H}\right]$ and monotonically decreases on $\left[v_{H},+\infty\right)$, and as the value of $v_{k}^{+}$increases continuously, $\varphi$ approaches the asymptote $\varphi=\tau$.

Proof. We first prove the domain of $\varphi$ is $[0,+\infty)$. Because $\left(u_{*}, v_{*}\right)$ in system (3) is globally asymptotically stable, and because $\mathrm{TH}<u_{*}$, any initial point from the $L_{3}$ will reach the impulse set $M$; so the definition domain of $\varphi$ is $[0,+\infty)$.

Next, we divide $L_{3}$ into two parts, which are $\left[0, v_{H}\right]$ and $\left[v_{H},+\infty\right)$. First of all, we can choose two points $Z_{a}\left(e^{-k_{1} D} \mathrm{TH}, v_{a}^{+}\right)$and $Z_{b}\left(e^{-k_{1} D} \mathrm{TH}, v_{b}^{+}\right)$and assume $0<v_{a}^{+}<v_{b}^{+}<v_{H}$, then the trajectory of these two points will intersect with $L_{4}$ at the two points $Z_{a+1}\left(\mathrm{TH}, v_{a+1}\right)$ and 


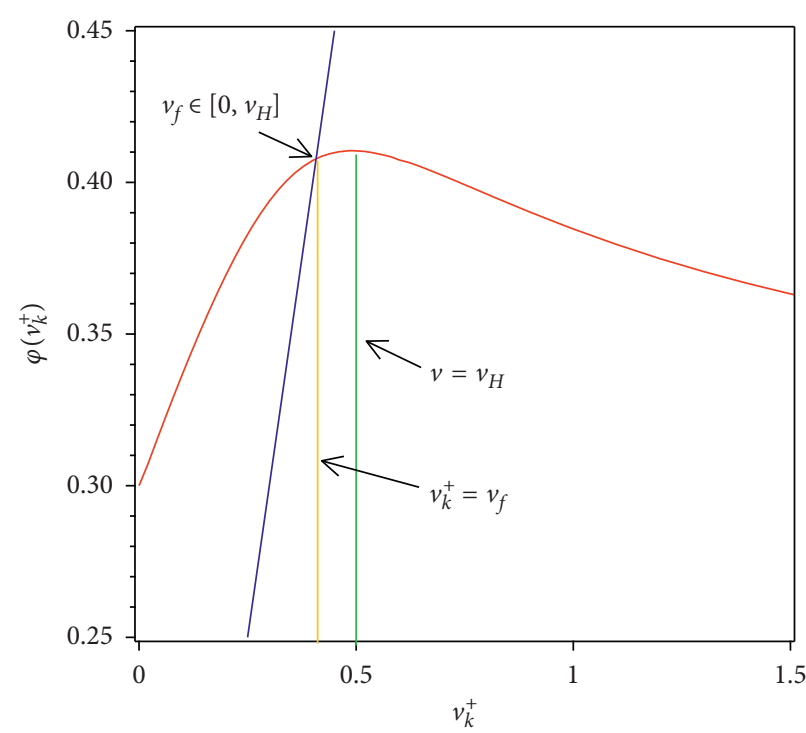

(a)

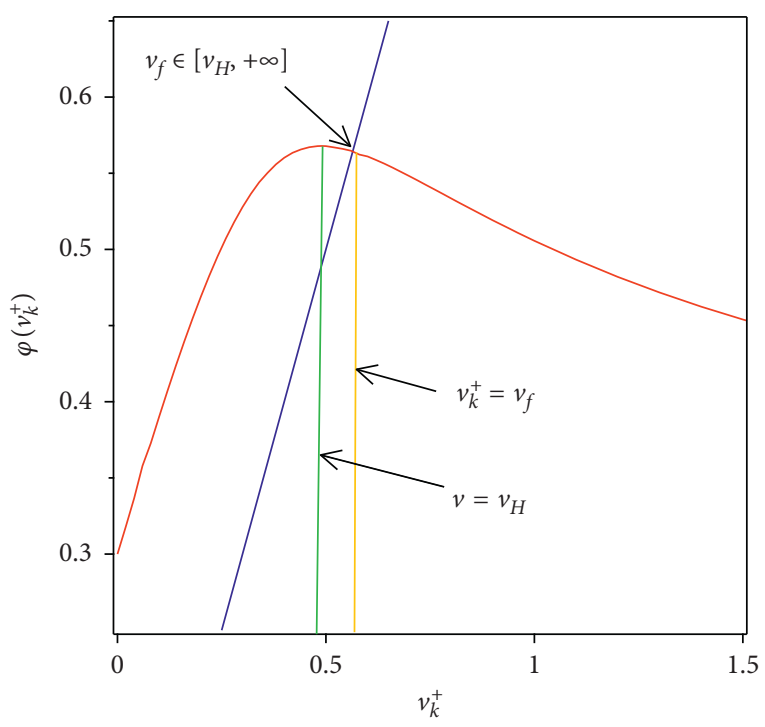

(b)

Figure 1: Poincaré map $\varphi$ related to the impulsive point series $v_{k}^{+}$with parameters fixed as $s=2, A=0.5, \delta=1, d=0.25, k_{1}=1, k_{2}=0.2$, $\mathrm{TH}=0.6$, and $\tau=0.3$. (a) $D=1$. (b) $D=0.5$.

$Z_{b+1}\left(\mathrm{TH}, v_{b+1}\right)$. According to Cauchy-Lipschitz theorem, $v_{a+1}<v_{b+1}$ is true for any $v_{a}^{+}<v_{b}^{+}$, and the formula of $\varphi$ is

$$
\begin{aligned}
& \varphi\left(v_{a}^{+}\right)=e^{-k_{2} D} \mathrm{TH} \cdot v_{a+1}+\tau, \\
& \varphi\left(v_{b}^{+}\right)=e^{-k_{2} D} \mathrm{TH} \cdot v_{b+1}+\tau .
\end{aligned}
$$

So it is easy to find for any $v_{a}^{+}$and $v_{b}^{+}$on $\left[0, v_{H}\right]$ and $v_{a}^{+}<v_{b}^{+}, \varphi\left(v_{a}^{+}\right)<\varphi\left(v_{b}^{+}\right)$is always true; therefore, $\varphi$ monotonically increases on $\left[0, v_{H}\right]$.

And then, we prove that the $\varphi$ is monotonically decreasing on $\left[v_{H},+\infty\right)$. Similarly, we take two numbers $v_{a}^{+}$ and $v_{b}^{+}$on $\left[v_{H},+\infty\right)$ and assume $v_{a}^{+}<v_{b}^{+}$, then the trajectories of the two initial points $Z_{a}\left(e^{-k_{1} D} \mathrm{TH}, v_{a}^{+}\right)$and $Z_{b}\left(e^{-k_{1} D} \mathrm{TH}, v_{b}^{+}\right)$from $L_{3}$ will cross the isoclinal line $L_{2}$ and then intersect $L_{3}$ at $Z_{a^{\prime}}\left(e^{-k_{1} D} \mathrm{TH}, v_{a^{\prime}}\right)$ and $Z_{b^{\prime}}\left(e^{-k_{1} D} \mathrm{TH}, v_{b^{\prime}}\right)$, respectively, where $v_{a^{\prime}}>v_{b^{\prime}}$. Then, these two curves will intersect $L_{4}$ at two points $Z_{a+1}\left(e^{-k_{1} D} \mathrm{TH}, v_{a+1}\right)$ and $Z_{b+1}\left(e^{-k_{1} D} \mathrm{TH}, v_{b+1}\right)$, respectively. According to CauchyLipschitz theorem, $v_{a+1}>v_{b+1}$ is always true, and the expression of $\varphi$ is obtained in (27), so for any $v_{a}^{+}<v_{b}^{+}$on $\left[v_{H},+\infty\right), \varphi\left(v_{a}^{+}\right)>\varphi\left(v_{b}^{+}\right)$is always true, and $\varphi$ is monotonically decreasing on $\left[v_{H},+\infty\right)$.

Then, we prove that as the value of $v_{k}^{+}$increases, $\varphi$ tends to be stable and approaches the asymptote $\varphi=\tau$. We define the closure of $\Omega_{1}$ as

$$
\Omega_{1}=\left\{(u, v) ; u>0, v>0, v<\frac{(1-A) u-u^{2}+A}{s+u-1}\right\} .
$$

Since $\varphi$ increases monotonically on $\left[0, v_{H}\right]$ and decreases monotonically on $\left[v_{H},+\infty\right), \Omega_{1}$ is the invariant set of system (5). Let

$$
L=v-\frac{(1-A) u-u^{2}+A}{s+u-1},
$$

if

$$
\left[(P(u, v), Q(u, v)) \cdot\left(\frac{u^{2}+2(s-1) u+(A-1) s+1}{(s+u-1)^{2}}, 1\right)\right]_{L=0} \leq 0 .
$$

Here $\cdot$ is the scalar product of two vectors, and then, the vector field will eventually reach the boundary $\Omega_{1}$, so $\Omega_{1}$ is an invariant set, and by calculation, one obtains

$$
\begin{aligned}
\left.V(u)\right|_{L=0} \doteq & \left(u(1-u)-\frac{s u v}{u+v+A}\right) \\
& \cdot \frac{u^{2}+2(s-1) u+(A-1) s+1}{(s+u-1)^{2}} \\
& +\delta v\left(\frac{u}{(u+v+A)}-d\right) \\
= & \delta v\left(\frac{u}{(u+v+A)}-d\right)<0 .
\end{aligned}
$$

Since $\varphi$ is monotonically increases on $\left[0, v_{H}\right]$ and monotonically decreases on $\left[v_{H},+E\right), \varphi\left(v_{0}^{+}\right)$is bounded for any $v_{0}^{+} \in\left[0, v_{H}\right]$ and $\varphi\left(\left[v_{H},+\infty\right)\right) \subset \varphi\left(\left[0, v_{H}\right]\right)$. From the Cauchy-Lipschitz Theorem, $v_{k+1}$ is only determined by $v_{k}^{+}$ and can be expressed by $v_{k+1}=\sigma\left(v_{k}^{+}\right)$. And any point on the phase set $N$ is always going to be $(\mathrm{d} v / \mathrm{d} t)<0$; hence, $\lim _{v_{k}^{+} \longrightarrow E} \sigma\left(v_{k}^{+}\right)=0$. Then, we have

$$
\lim _{v_{k}^{+} \longrightarrow E} \varphi\left(v_{k}^{+}\right)=\lim _{v_{k}^{+} \longrightarrow E} e^{-k_{2} D} \sigma\left(v_{k}^{+}\right)+\tau=\tau \text {. }
$$

So as the value of $v_{k}^{+}$increases, $\varphi$ tends to be stable and approaches the asymptote $\varphi=\tau$.

Since $\varphi$ increases monotonically on $\left[0, v_{H}\right]$ and decreases monotonically on $\left[v_{H},+E\right) \subset \varphi\left(\left[0, v_{H}\right]\right), \varphi\left(\left[v_{H},+E\right)\right)$; thus, $\varphi$ takes the maximum value at $v_{H}$ and takes the minimum value at 0 , where $\varphi\left(v_{H}\right)=v\left(e^{-k_{1} D} \mathrm{TH}, v_{H}\right)+\tau$ and $\varphi(0)=\tau$, so the range of value is $\left[\tau, \varphi\left(v_{H}\right)\right]$. 
Theorem 2. $\varphi$ is continuously differentiable.

Proof. Here, we can use the initial conditions of continuity and differentiability theorem which is to take parameters of Cauchy theorem and Lipschitz theorem to determine the continuity and differentiability of $\varphi$; by system (5), we can get that both $P(u, v)$ and $Q(u, v)$ functions are continuously differentiable in the first quadrant; so by Cauchy theorem and Lipschitz theorem with parameters, we can get that the $\varphi$ is a continuously differentiable function.

Theorem 3. $\varphi$ always has at least one fixed point if $\tau>0$.

Proof. From Theorem 1, we know that $\varphi$ is monotonically increasing on $\left[0, v_{H}\right]$ and monotonically decreasing on $\left[v_{H},+\infty\right)$. Then, we divide it into two cases to discuss the existence of the fixed point for $\varphi$.

Case I: when $\varphi\left(v_{H}\right)<v_{H}$, on the one hand, $\varphi(0)=\tau>0$, so $\varphi$ has at least one number $v_{c}$ on $\left[0, v_{H}\right)$ such that $\varphi\left(v_{c}\right)=v_{c}$; on the other hand, since $\varphi$ is monotonically decreasing on $\left[v_{H},+E\right)$ and $\varphi\left(v_{H}\right)<v_{H}$, $\varphi$ has no fixed point on $\left[v_{H},+E\right)$. In conclusion, when $\varphi\left(v_{H}\right)<v_{H}$, the $\varphi$ has at least one fixed point.

Case II: when $\varphi\left(v_{H}\right) \geq v_{H}$, because $\varphi$ monotonically decreases on $\left[v_{H},+E\right)$ and as the value of $v_{k}^{+}$increases continuously, $\varphi$ approaches asymptote $\varphi=\tau$, so $\varphi$ has only one point $v_{c}$ on $\left[v_{H},+E\right)$ such that $\varphi\left(v_{c}\right)=v_{c}$. And the number of fixed points on $\left[0, v_{H}\right)$ is unknown. So when $\varphi\left(v_{H}\right) \geq v_{H}$, the $\varphi$ has at least one fixed point.

In conclusion, $\varphi$ always has at least one fixed point.

\section{Study on the Periodic Solutions of System (5)}

4.1. Boundary Periodic Solutions of System (5). For system (5), if the predator population becomes extinct and the predator also terminates its release, then system (5) has a boundary periodic solution, which produces the following system:

$$
\left\{\begin{array}{l}
u^{\prime}(t)=u(1-u), u(t)<\mathrm{TH}, \\
u\left(t^{+}\right)=e^{-k_{1} D} u(t), u(t)=\mathrm{TH}
\end{array}\right.
$$

Solving (34) with initial value $u\left(0^{+}\right)=e^{-k_{1} D} \mathrm{TH}$,

$$
u(t)=\frac{e^{-k_{1} D} \mathrm{TH} e^{t}}{1+e^{-k_{1} D} \mathrm{TH} e^{t}-e^{-k_{1} D} \mathrm{TH}} .
$$

The trajectory from the initial point will eventually intersect with the straight line of the impulse set over time:

$$
\mathrm{TH}=\frac{e^{-k_{1} D} \mathrm{TH} e^{T}}{1+e^{-k_{1} D} \mathrm{TH} e^{T}-e^{-k_{1} D} \mathrm{TH}} .
$$

Solving the equation of $T$ and $D$, we get

$$
\begin{aligned}
& T=\ln \frac{1-e^{-k_{1} D} \mathrm{TH}}{e^{-k_{1} D}(1-\mathrm{TH})}, \\
& D=\frac{1}{k_{1}} \ln \left(\mathrm{TH}+e^{T}(1-\mathrm{TH})\right),
\end{aligned}
$$

where $T$ is the period of the boundary periodic solution and $D$ is the insecticide dose required to control the number of pests below the TH. Then, the boundary periodic solution of system (5) with a period of $T$ is

$$
\left\{\begin{array}{l}
u(t)=\frac{\mathrm{TH} e^{(t-(k-1) T)}}{\mathrm{TH}-\mathrm{TH} e^{(t-(k-1) T)}-e^{-k_{1} D}} \\
v(t)=0
\end{array}\right.
$$

Theorem 4. The boundary periodic solution $\left(U^{T}(t), 0\right)$ of system (5) is asymptotically stable if

$$
\begin{aligned}
R_{1}= & \mid \frac{e^{-k_{1} D} e^{-k_{2} D}\left(1-e^{-k_{1} D} \mathrm{TH}\right)}{1-\mathrm{TH}} \exp \left(\int_{0}^{T}\right. \\
& {\left[1-2 u^{T}(t)-\delta d+\frac{\delta u^{T}(t)}{u^{T}(t)+v^{T}(t)+A}\right.} \\
& \left.\left.+\frac{s v^{T}(t)\left(v^{T}(t)+A\right)+\delta u^{T}(t) v^{T}(t)}{\left(u^{T}(t)+v^{T}(t)+A\right)^{2}}\right] \mathrm{~d} t\right) \mid<1 .
\end{aligned}
$$

Proof. By Definition 3, we obtain

$$
\begin{aligned}
P(u, v) & =u(1-u)-\frac{s u v}{(u+v+A)}, \\
Q(u, v) & =\delta v\left(\frac{u}{(u+v+A)}-d\right), \\
\alpha(u, v) & =u\left(e^{-k_{1} D}-1\right), \\
\beta(u, v) & =v\left(e^{-k_{2} D}-1\right)+\tau, \\
\phi(u, v) & =u-\mathrm{TH}, \\
\left(u^{T}(T), v^{T}(T)\right) & =(\mathrm{TH}, 0), \\
\left(u^{T}\left(T^{+}\right), v^{T}\left(T^{+}\right)\right) & =\left(e^{-k_{1} D} \mathrm{TH}, 0\right) .
\end{aligned}
$$

From the above formula, we can get

$$
\begin{aligned}
& \frac{\partial P}{\partial u}=1-2 u-\frac{s v(v+A)}{(u+v+A)^{2}}, \\
& \frac{\partial Q}{\partial v}=\delta\left(\frac{u}{u+v+A}-\frac{u v}{(u+v+A)^{2}}-d\right), \\
& \frac{\partial \alpha}{\partial u}=e^{-k_{1} D}-1, \\
& \frac{\partial \beta}{\partial v}=e^{-k_{2} D}-1,
\end{aligned}
$$

$$
\begin{aligned}
& \frac{\partial \phi}{\partial u}=1, \\
& \frac{\partial \alpha}{\partial v}=\frac{\partial \beta}{\partial u}=\frac{\partial \phi}{\partial v}=0,
\end{aligned}
$$


so

$$
\begin{aligned}
\Delta_{1} & =\frac{P_{+}((\partial \beta / \partial v)(\partial \phi / \partial u)-(\partial \beta / \partial u)(\partial \phi / \partial v)+(\partial \phi / \partial u))+Q_{+}((\partial \alpha / \partial u)(\partial \phi / \partial v)-(\partial \alpha / \partial v)(\partial \phi / \partial u)+(\partial \phi / \partial u))}{P(\partial \phi / \partial u)+Q(\partial \phi / \partial v)} \\
& =\frac{e^{-k_{1} D} e^{-k_{2} D}\left(1-e^{-k_{1} D} \mathrm{TH}\right)}{1-\mathrm{TH}}
\end{aligned}
$$

In addition,

$$
\begin{gathered}
\exp \left(\int_{0}^{T}\left[\frac{\partial P}{\partial u}\left(u^{T}(t), v^{T}(t)\right)+\frac{\partial Q}{\partial v}\left(u^{T}(t), v^{T}(t)\right)\right] \mathrm{d} t\right) \\
=\exp \left(\int _ { 0 } ^ { T } \left[1-2 u^{T}(t)-\delta d+\frac{\delta u^{T}(t)}{u^{T}(t)+v^{T}(t)+A}\right.\right. \\
\left.\left.+\frac{s v^{T}(t)\left(v^{T}(t)+A\right)+\delta u^{T}(t) v^{T}(t)}{\left(u^{T}(t)+v^{T}(t)+A\right)^{2}}\right] \mathrm{~d} t\right) .
\end{gathered}
$$

The expression of $\mu_{1}$ is

$$
\begin{aligned}
\mu_{1}= & \Delta_{1} \exp \left(\int_{0}^{T}\left[\frac{\partial P}{\partial u}\left(u^{T}(t), v^{T}(t)\right)+\frac{\partial Q}{\partial v}\left(u^{T}(t), v^{T}(t)\right)\right] \mathrm{d} t\right) \\
= & \frac{e^{-k_{1} D} e^{-k_{2} D}\left(1-e^{-k_{1} D} \mathrm{TH}\right)}{1-\mathrm{TH}} \exp \left(\int _ { 0 } ^ { T } \left[1-2 u^{T}(t)-\delta d\right.\right. \\
& \left.\left.+\frac{\delta u^{T}(t)}{u^{T}(t)+v^{T}(t)+A}+\frac{s v^{T}(t)\left(v^{T}(t)+A\right)+\delta u^{T}(t) v^{T}(t)}{\left(u^{T}(t)+v^{T}(t)+A\right)^{2}}\right] \mathrm{~d} t\right) .
\end{aligned}
$$

If condition (39) is true, then $\left|\mu_{1}\right|<1$. It means the periodic solution $\left(u^{T}(t), 0\right)$ of the boundary is asymptotically stable.

4.2. Existence and Stability of Periodic Solutions of Order$k(k \geq 1)$ at $\tau>0$. From Theorem 3, the Poincaré map function $\varphi$ of system (5) has at least one fixed point; that is to say, system (5) must have at least one order-1 periodic solution.

Theorem 5. The order-1 periodic solution $(\xi(t), \eta(t))$ is orbitally asymptotically stable if and only if

$$
\left|\frac{e^{-k_{2} D} e^{-k_{1} D}\left(1-e^{-k_{1} D} \mathrm{TH}\right)-\left(s e^{-k_{1} D}\left(e^{-k_{2} D} \eta_{0}+\tau\right) /\left(e^{-k_{1} D} \mathrm{TH}+e^{-k_{2} D} \eta_{0}+\tau+A\right)\right)}{1-\mathrm{TH}-\left(s \eta_{0} /\left(\mathrm{TH}+\eta_{0}+A\right)\right)} \cdot \exp \left(\int_{0}^{T} U(t) \mathrm{d} t\right)\right|<1,
$$

where

$$
\begin{aligned}
U(t)= & \exp \left(\int _ { 0 } ^ { T } \left[1-2 \xi(t)-\delta d+\frac{\delta \xi(t)}{\xi(t)+\eta(t)+A}\right.\right. \\
& \left.\left.+\frac{s \eta(t)(\eta(t)+A)+\delta \xi(t) \eta(t)}{(\xi(t)+\eta(t)+A)^{2}}\right] d t\right) .
\end{aligned}
$$

Proof. We use $R\left(\mathrm{TH}, \eta_{0}\right)$ and $R^{+}\left(e^{-k_{1} D} \mathrm{TH}, e^{-k_{2} D} \eta_{0}+\tau\right)$ to represent the start point and the endpoint of the order-1 periodic solution, respectively. From Theorem 4, we know that the Floquet multiplier

$$
\begin{aligned}
\mu_{1} & =\Delta_{1} \exp \left(\int_{0}^{T}\left[\frac{\partial P}{\partial u}\left(\xi^{T}(t), \eta^{T}(t)\right)+\frac{\partial Q}{\partial v}\left(\xi^{T}(t), \eta^{T}(t)\right)\right] \mathrm{d} t\right) \\
& =\frac{e^{-k_{2} D} e^{-k_{1} D}\left(1-e^{-k_{1} D} \mathrm{TH}\right)-\left(s e^{-k_{1} D}\left(e^{-k_{2} D} \eta_{0}+\tau\right) /\left(e^{-k_{1} D} \mathrm{TH}+e^{-k_{2} D} \eta_{0}+\tau+A\right)\right)}{1-T H-\left(s \eta_{0} /\left(\mathrm{TH}+\eta_{0}+A\right)\right)} \cdot \exp \left(\int_{0}^{T} U(t) \mathrm{d} t\right) .
\end{aligned}
$$

If (45) is true, then $\left|\mu_{1}\right|<1$, so the order-1 periodic solution is always orbitally asymptotically stable.
Theorem 6. If $\varphi\left(v_{H}\right)<v_{H}$, there is at least one locally asymptotically stable order-1 periodic solution in system (5). 
Furthermore, if there is only one fixed point on $\left[0, v_{H}\right]$, then there is a globally asymptotically stable order-1 periodic solution of system (5).

Proof. If $\varphi\left(v_{H}\right)<v_{H}$, Poincaré map $\varphi$ has at least one fixed point. This also proves that there is at least one order-1 periodic solution in system (5). From Theorem 5, we know that the periodic solution is always asymptotic stable if $\left|\mu_{1}\right|<1$. So if $\varphi\left(v_{H}\right)<v_{H}$, then there is at least one locally asymptotically stable order-1 periodic solution in system (5).

If $\varphi\left(v_{H}\right)<v_{H}$, there is only one fixed point on $\left[0, v_{H}\right]$. This proves that there is a unique order-1 periodic solution in system (5). According to Theorem 5, we can conclude that the periodic solution is asymptotic stable.

For any trajectory starting from $\left(e^{-k_{1} D} \mathrm{TH}, v_{0}^{+}\right)$, if $v_{0}^{+} \in\left[0, v_{H}\right]$, then $v_{0}^{+}<\varphi\left(v_{0}^{+}\right)<v_{H}$. After $n$ times pulses, $\varphi^{n}\left(v_{0}^{+}\right)$monotonically increases, so $\lim _{n \longrightarrow+\infty} \varphi^{n}\left(v_{0}^{+}\right)=v_{H}$.

In contrary, if $v_{0}^{+}>v_{H}$, we need to discuss this according to different cases. On the one hand, if $\varphi^{n}\left(v_{0}^{+}\right)>v_{H}$ is always holding, we can conclude that $\varphi^{n}\left(v_{0}^{+}\right)$is monotonically decreasing because $\varphi^{n}\left(v_{0}^{+}\right)<v_{0}^{+}$and $\lim _{n \longrightarrow+\infty} \varphi^{n}\left(v_{0}^{+}\right)=v_{H}$. On the other hand, $\varphi^{n}\left(v_{0}^{+}\right)>v_{H}$ is not true for all $n$. We make $n_{0}$ the smallest which satisfies $\varphi^{n_{0}}\left(v_{0}^{+}\right)<v_{H}$. Then, there must be a positive integer $n_{1}>n_{0}$ and $\varphi^{n_{1}}\left(v_{0}^{+}\right)$monotonically increases as $n_{1}$ increases, so $\lim _{n_{1} \longrightarrow+\infty} \varphi^{n}\left(v_{0}^{+}\right)=v_{H}$. Therefore, there is a globally asymptotically stable order-1 periodic solution of system (5).

Theorem 7. If $\varphi\left(v_{H}\right)>v_{H}, \varphi^{2}\left(v_{H}\right) \geq v_{H}$, and there are no fixed points on $\left[0, v_{H}\right]$ of $\varphi$; then, system (5) either has a stable order-1 periodic solution or a stable order-2 periodic solution.

Proof. If there are no fixed points on $\left[0, v_{H}\right]$ of $\varphi$, then there is a positive constant $i$ which makes $v_{i}^{+} \leq v_{H}$ and $v_{i+1}^{+} \geq v_{H}$. Based on the definition of the Poincaré map $\varphi$, we get $v_{i+1}^{+}=$ $\varphi\left(v_{i}^{+}\right) \leq \varphi\left(v_{H}\right)$ and $v_{i+1}^{+} \in\left[v_{H}, \varphi\left(v_{H}\right)\right]$. Because the $\varphi$ is decreasing on $\left[v_{H},+\infty\right)$ for any $v_{1}^{+}>v_{H}$, after one pulse, there is $v_{2}^{+}=\varphi\left(v_{1}^{+}\right) \leq \varphi\left(v_{H}\right)$. Therefore, $v_{i+1}^{+} \in\left[v_{H}, \varphi\left(v_{H}\right)\right]$ holds for all $i \geq 2$. Also, $\varphi^{2}$ is monotonically increasing on $\left[v_{H},+\infty\right)$ so that

$$
\varphi\left(\left[v_{H}, \varphi\left(v_{H}\right)\right]\right)=\left[\varphi^{2}\left(v_{H}\right), \varphi\left(v_{H}\right)\right] \subset\left[v_{H}, \varphi\left(v_{H}\right)\right] .
$$

Next, the existence of the periodic solution is discussed. First of all, for any $v_{0}^{+} \in\left[v_{H}, \varphi\left(y_{H}\right)\right]$ and make $v_{1}^{+}=\varphi\left(v_{0}^{+}\right) \neq v_{0}^{+}$and $v_{2}^{+}=\varphi^{2}\left(v_{0}^{+}\right) \neq v_{0}^{+}$. If $v_{1}^{+}=\varphi\left(v_{0}^{+}\right)=v_{0}^{+}$and $v_{2}^{+}=\varphi^{2}\left(v_{0}^{+}\right)=v_{0}^{+}$, then $v_{0}^{+}$is the fixed point of $\varphi$ which proves system (5) either has a stable order-1 periodic solution or a stable order-2 periodic solution. So the relations about $v_{H}$, $\varphi\left(v_{H}\right), v_{0}^{+}, v_{1}^{+}$, and $v_{2}^{+}$are needed to be discussed.

(i) $v_{H} \leq v_{2}^{+}<v_{0}^{+}<v_{1}^{+} \leq \varphi\left(v_{H}\right)$ (Figure 2(a)). Then, $v_{3}^{+}=$ $\varphi\left(v_{2}^{+}\right)>\varphi\left(v_{0}^{+}\right)=v_{1}^{+}$and $v_{4}^{+}=\varphi\left(v_{3}^{+}\right)>\varphi\left(v_{1}^{+}\right)=v_{2}^{+}$. It can be obtained by mathematical induction that

$$
\begin{aligned}
v_{H} & \leq \cdots<v_{2 n+2}^{+}<v_{2 n}^{+}<\cdots<v_{2}^{+}<v_{0}^{+} \\
& <v_{1}^{+}<\cdots<v_{2 n-1}^{+}<v_{2 n+1}^{+}<\cdots \leq \varphi\left(v_{H}\right) .
\end{aligned}
$$

(ii) $v_{H} \leq v_{0}^{+}<v_{2}^{+}<v_{1}^{+} \leq \varphi\left(v_{H}\right)$ (Figure 2(b)). Then, $v_{1}^{+}=$ $\varphi\left(v_{0}^{+}\right)>\varphi\left(v_{2}^{+}\right)=v_{3}^{+}>v_{2}^{+}=\varphi\left(v_{1}^{+}\right)$and $v_{2}^{+}=\varphi\left(v_{1}^{+}\right)<\varphi$ $\left(v_{3}^{+}\right)=v_{4}^{+}<v_{3}^{+}=\varphi\left(v_{2}^{+}\right)<v_{1}^{+}$; i.e., it can be obtained by mathematical induction that

$$
\begin{aligned}
v_{H} & \leq v_{0}^{+}<v_{2}^{+}<\cdots<v_{2 n}^{+}<v_{2 n+2}^{+} \\
& <\cdots<v_{2 n+1}^{+}<v_{2 n-1}^{+}<\cdots<v_{1}^{+} \leq \varphi\left(v_{H}\right) .
\end{aligned}
$$

(iii) $v_{H} \leq v_{1}^{+}<v_{2}^{+}<v_{0}^{+} \leq \varphi\left(v_{H}\right)$ (Figure 2(c)). In the same way, about case (ii), we can obtain

$$
\begin{aligned}
v_{H} & \leq v_{1}^{+}<\cdots<v_{2 n-1}^{+}<v_{2 n+1}^{+} \\
& <\cdots<v_{2 n+2}^{+}<v_{2 n}^{+}<\cdots<v_{2}^{+}<v_{0}^{+} \leq \varphi\left(v_{H}\right) .
\end{aligned}
$$

(iv) $v_{H} \leq v_{1}^{+}<v_{0}^{+}<v_{2}^{+} \leq \varphi\left(v_{H}\right)$ (Figure 2(d)). By using the same method as case (i), we can obtain

$$
\begin{aligned}
v_{H} & \leq \cdots<v_{2 n+1}^{+}<v_{2 n-1}^{+}<\cdots<v_{1}^{+}<v_{0}^{+} \\
& <v_{2}^{+}<\cdots<v_{2 n}^{+}<v_{2 n+2}^{+}<\cdots \leq \varphi\left(v_{H}\right) .
\end{aligned}
$$

For case (ii), $\varphi^{2 n}\left(v_{0}^{+}\right)=v_{2 n}^{+}$is monotonically increasing and $\varphi^{2 n+1}\left(v_{0}^{+}\right)=v_{2 n+1}^{+}$is monotonically decreasing; for case (iii), $\varphi^{2 n}\left(v_{0}^{+}\right)=v_{2 n}^{+}$is monotonically decreasing and $\varphi^{2 n+1}\left(v_{0}^{+}\right)=v_{2 n+1}^{+}$is monotonically increasing. It is concluded that for case (ii) and case (iii), there exists either a unique fixed point $v_{a}$ such that

$$
\lim _{n \longrightarrow \infty} v_{2 n}^{+}=\lim _{n \longrightarrow \infty} v_{2 n+1}^{+}=v_{a}, \quad v_{a} \in\left[v_{H}, \varphi\left(v_{H}\right)\right],
$$

or exists two distinct values $v_{a}$ and $v_{b}$ and $v_{a} \neq v_{b}$ such that

$$
\begin{array}{ll}
\lim _{n \longrightarrow \infty} v_{2 n}^{+}=v_{a}, & v_{a} \in\left[v_{H}, \varphi\left(v_{H}\right)\right], \\
\lim _{n \longrightarrow \infty} v_{2 n+1}^{+}=v_{b}, & v_{b} \in\left[v_{H}, \varphi\left(v_{H}\right)\right] .
\end{array}
$$

However, for cases (i) and (iv), only the later case can be true.

These results verify that there exists either an order-1 periodic solution or a periodic solution+ (5).

Theorem 8. If $\varphi\left(v_{H}\right)>v_{H}$, then $v_{m}^{+}$satisfies $v_{m}^{+}=\min \left\{v^{+}: \varphi_{m}\left(v^{+}\right)=v^{+}\right\}$, and if there is no fixed point on $\left(0, v_{H}\right)$, when $\varphi^{2}\left(v_{H}\right) \geq v_{m}^{+}$, then system (5) has an order-3 periodic solution.

Proof. If $\varphi\left(v_{H}\right)>v_{H}$, and there is no fixed point on $\left(0, v_{H}\right)$, it can be seen from Theorem 1 that there is a unique order-1 period solution in $\left(v_{H}, \varphi\left(v_{H}\right)\right)$ :

$$
\varphi(\widetilde{u})=\widetilde{u}, \quad \widetilde{u} \in\left(v_{H}, \varphi\left(v_{H}\right)\right),
$$

because Poincaré map $\varphi$ is continuous on closed intervals $[0, \tilde{u}]$ and

$$
\begin{aligned}
& \varphi(0)=\tau, \\
& \varphi(\tilde{u})=\widetilde{u} .
\end{aligned}
$$




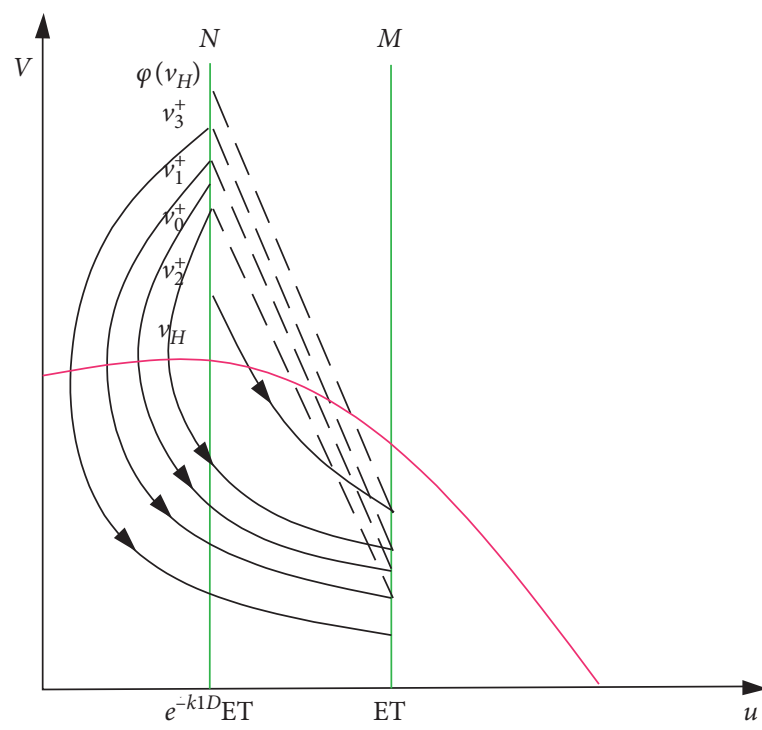

(a)

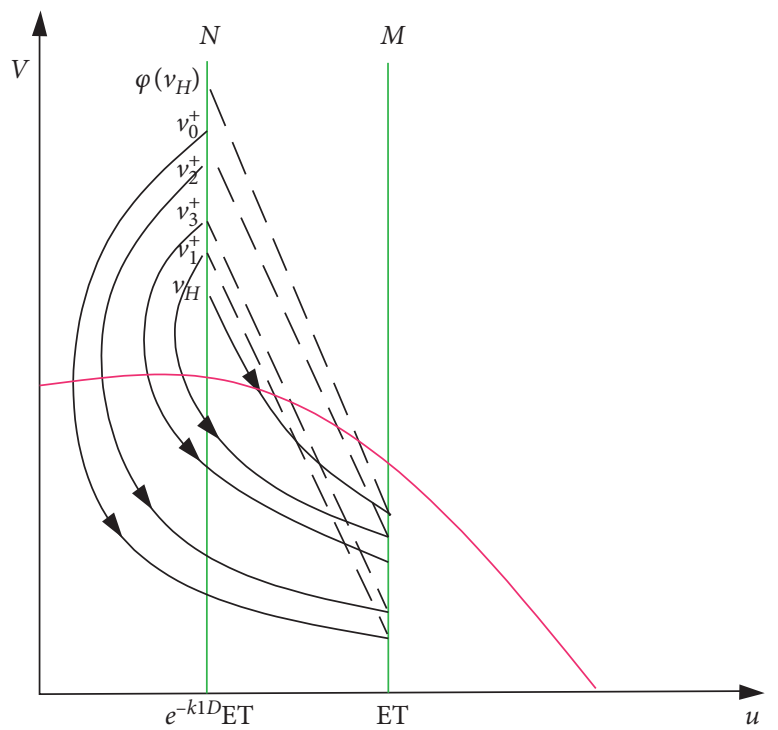

(c)

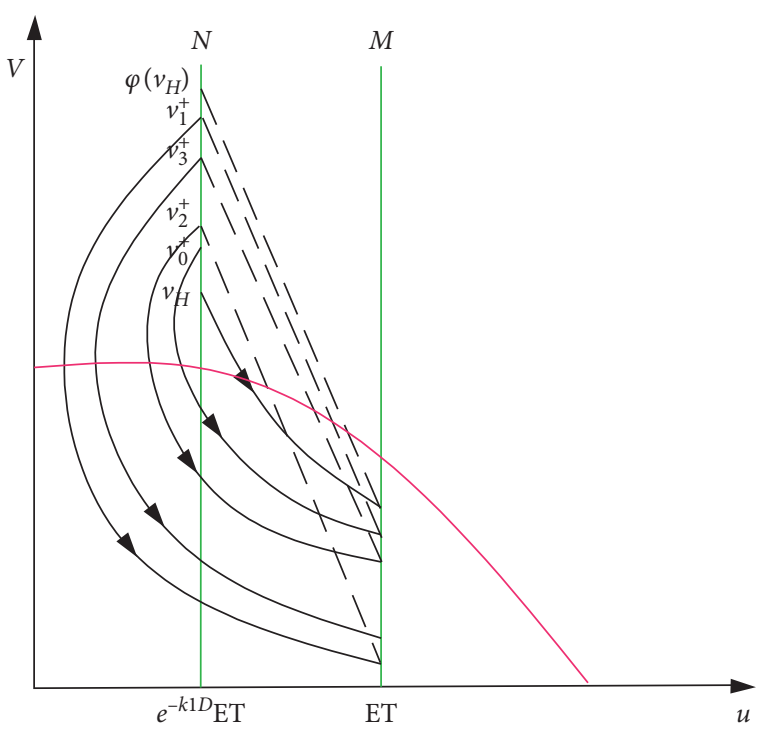

(b)

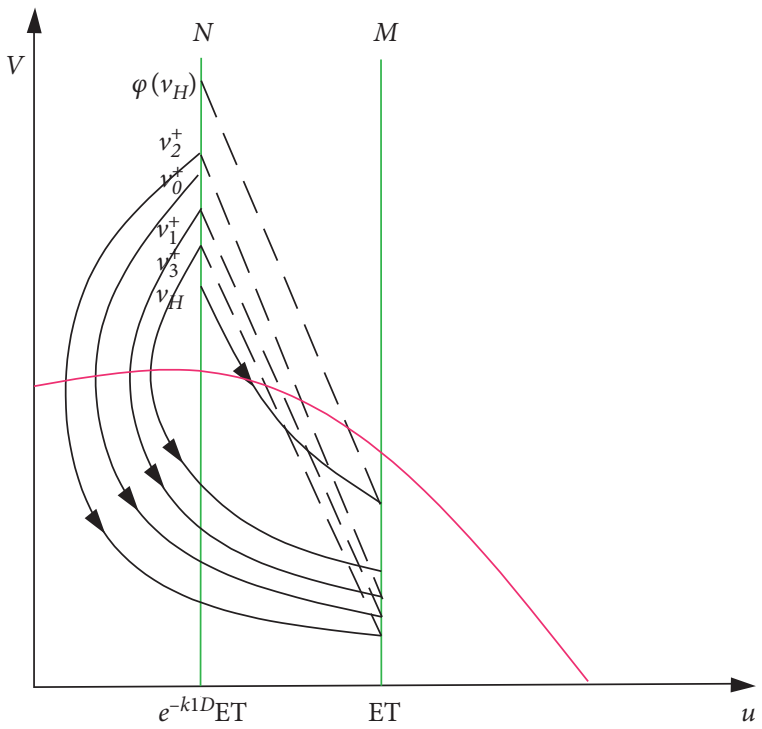

(d)

FIgURE 2: Four cases of existence of periodic solutions in Theorem 7.

According to the intermediate value theorem, there exists $v_{m}^{+} \in(0, \widetilde{u})$, and $\varphi\left(v_{m}^{+}\right)=u_{H}$.

Furthermore,

$$
\begin{aligned}
\varphi^{3}\left(v_{m}^{+}\right) & =\varphi^{2}\left(u_{H}\right)<v_{m}^{+}, \\
\varphi^{3}(0) & >0 .
\end{aligned}
$$

According to the properties of continuous functions on closed intervals, there must be at least one value of $\vec{u}$ to enable

$$
\varphi^{3}(\vec{u})=\vec{u} .
$$

This means that system (5) has an order-3 periodic solution.

If we replace condition $\varphi^{2}\left(v_{H}\right) \geq v_{m}^{+}$in Theorem 8 with condition $\varphi^{k-1}\left(u_{H}\right)<v_{m}^{+}$, where $\varphi\left(v_{m}^{+}\right)=u_{H}$, the order- $k$ periodic solution of system (5) can be obtained by a similar method of Theorem 8 .

\section{Numerical Simulation}

In the state impulse feedback control, we assign appropriate thresholds for TH and $D$ (see Figures 3 and 4). In Figures 3 and 4 , the red line shows the trajectory of the system without the impulse, and the green line shows the trajectory of the system with the impulse; this suggests that populations of predators and pests can be kept within a stable range.

It can be seen from Figure 5 that different initial points will eventually converge to the same order-1 periodic solution and tend to be stable; this indicates the global asymptotic stability of the order-1 periodic solution. 


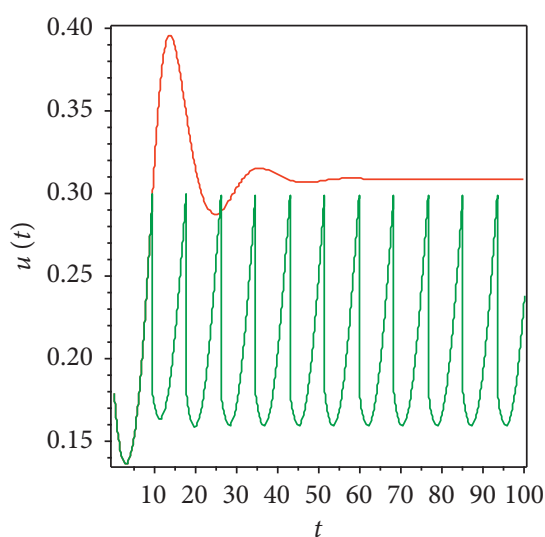

(a)

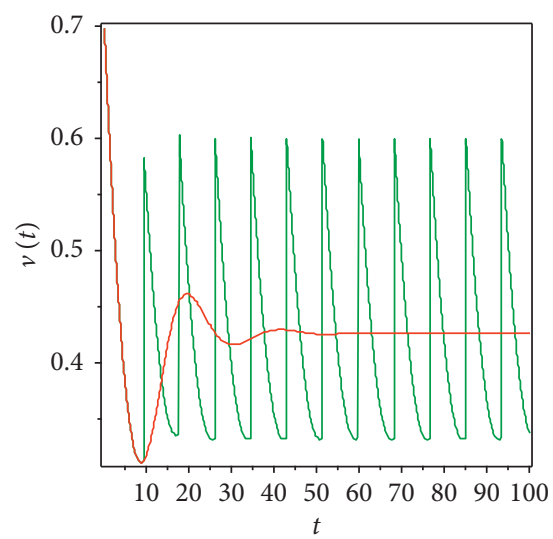

(b)

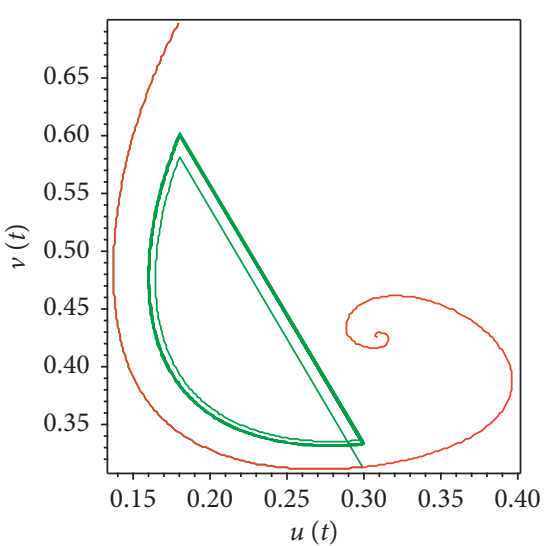

(c)

Figure 3: State pulse feedback control strategy of system (5): $s=2, A=0.5, \delta=1, d=0.25, k_{1}=1, k_{2}=0.2, \mathrm{TH}=0.6, \tau=0.3$, and $D=0.5$.

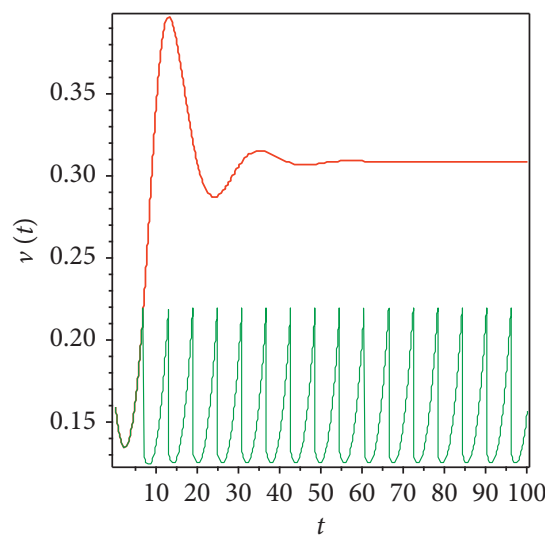

(a)

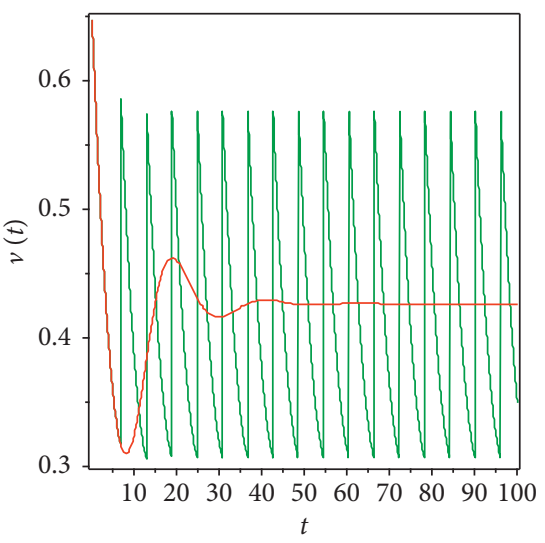

(b)

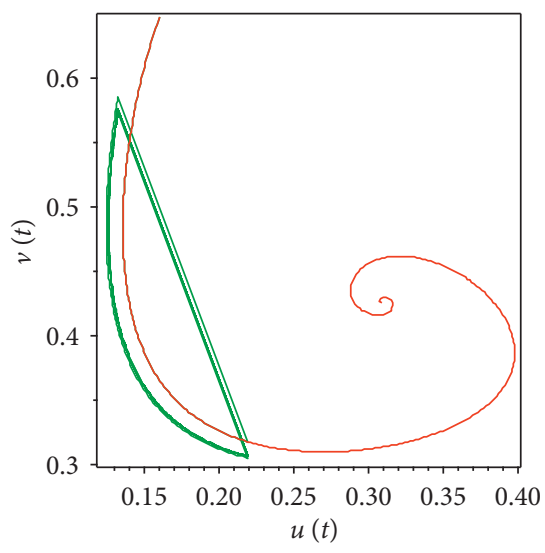

(c)

Figure 4: State pulse feedback control strategy of system (5): $s=2, A=0.5, \delta=1, d=0.25, k_{1}=1, k_{2}=0.2, \mathrm{TH}=0.6, \tau=0.3$, and $D=1$.

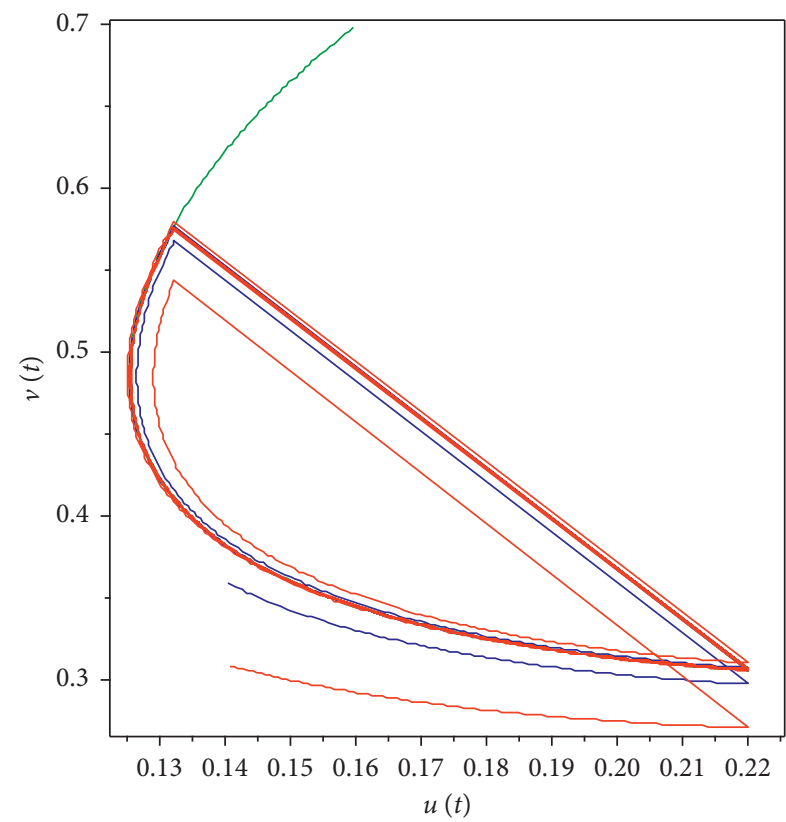

FIGURE 5: State pulse feedback control strategy of system (5): $s=2$, $A=0.5, \delta=1, d=0.25, k_{1}=1, k_{2}=0.2, \mathrm{TH}=0.6, \tau=0.3$, and $D=1$, and with three different initial points.
The above numerical simulation also shows that the number of pests can be controlled in the state pulse feedback control, which verifies the feasibility of state pulse feedback control.

In Section 4.1, when the predators disappear and the pests reach $\mathrm{TH}$, we obtain the expression of the boundary period solution and the expression of the pesticide dose. Next, we discuss which key factors can affect the pesticide dose $D$. We gave some reasonable parameters, as shown in Figure 6 . The results show that as $e^{-k_{1} D} \mathrm{TH}$ decreases, dose $D$ must also be increased (Figure 6(a)). Furthermore, as the $T$ of chemical control increases, the dose $D$ increases (Figure 6(b)). Biologically, we need to consider both the threshold TH and the period $T$ in the process of pest control.

According to condition (39), we can judge whether the chemical control can stabilize the boundary periodic solution alone. $R_{1}<1$ means that chemical control by $D$ dose alone can control the pest population below the TH, and vice versa. Therefore, how much the dose $D$ and the threshold TH can affect $R_{1}$ has drawn our attention. For these, we have carried out numerical simulations, as shown in Figure 7(a). The results show that when a single chemical control method is used, high dose $D$ can control the pest 


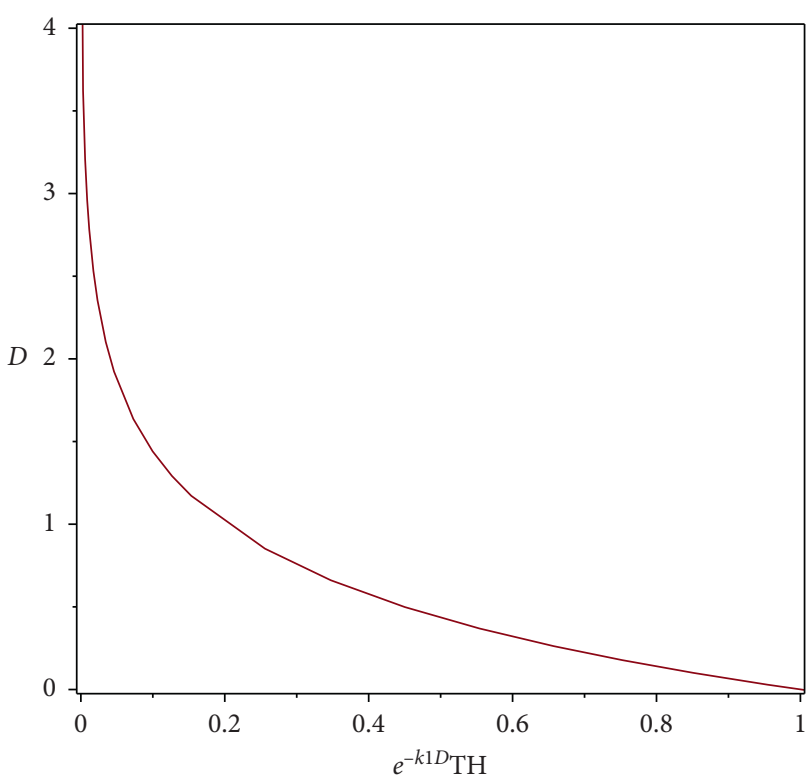

(a)

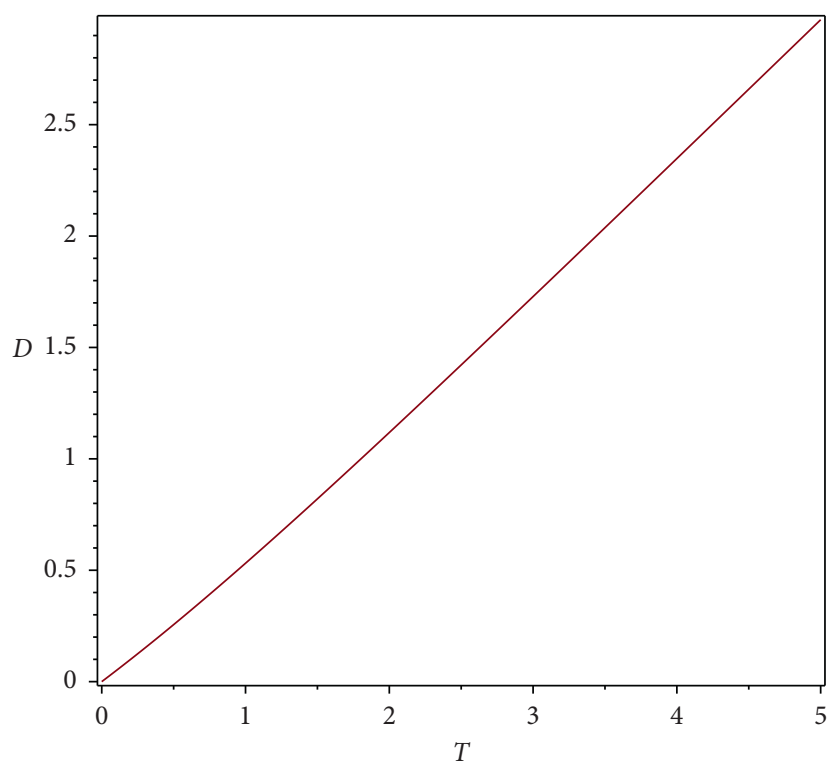

(b)

FIgURE 6: Effects of parameters $e^{-k_{1} D} \mathrm{TH}$ and $T$ on the $D$ of chemical control: (a) $T=20, k_{1}=1.6$; (b) $\mathrm{TH}=0.22, k_{1}=1.6$.

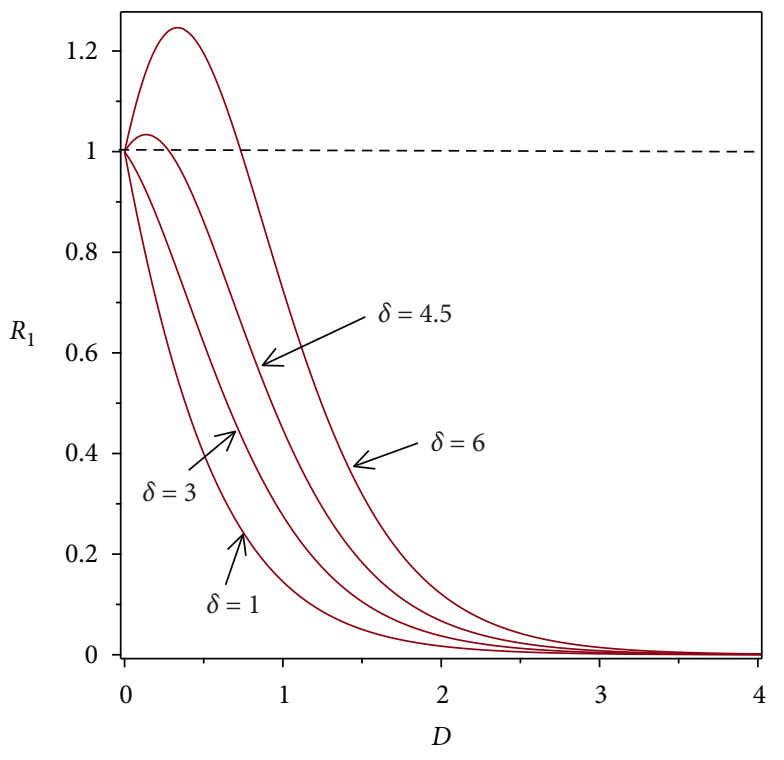

(a)

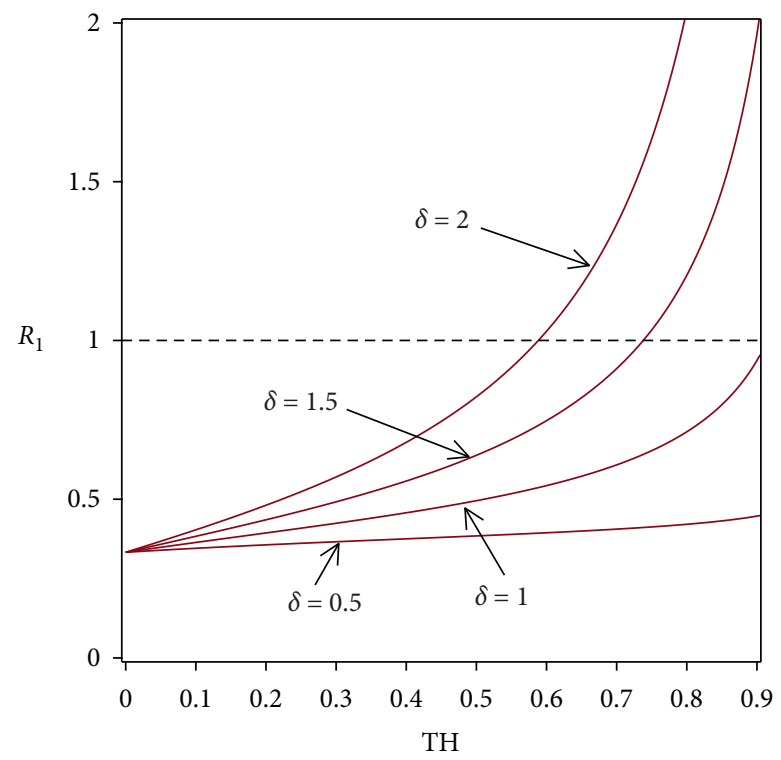

(b)

Figure 7: Effects of control parameters on the threshold condition $R_{1}$ and $k_{1}=-1.6, k_{2}=-1.8, d=0.25$, and $A=0.5$ : (a) TH=0.22; (b) $D=0.5$.

population. In addition, as shown in Figure $7(\mathrm{~b})$, for a relatively small TH, we have $R_{1}<1$, and once TH is greater than a certain value, $R_{1}>1$. The results show that under the fixed parameter values, the smaller the TH value, the better the prevention and control of pests. In the process of pesticide management, as long as we choose a reasonable threshold $\mathrm{TH}$ under pulse state feedback control, we can avoid excessive use of pesticides and reduce some negative effects of pesticides.

\section{Conclusion}

Compared with previous studies on state-dependent feedback control, we mainly do the following work: the global dynamics of complex models are studied according to the Poincaré map, and the main properties of Poincaré map are studied to prove the existence of fixed points and the existence of order- $k(k \geq 1)$ periodic solutions. Besides, we study the effect of pesticide dose on single chemical control 
or chemical control combined with biological control. The results show that the pest population density can not only be controlled below the threshold under the state pulse feedback control but also avoid excessive application of pesticides and reduce some negative effects of pesticides.

\section{Data Availability}

The data used to support the findings of this study are available upon request to the corresponding author.

\section{Conflicts of Interest}

The authors declare that they have no conflicts of interest.

\section{Acknowledgments}

This work was supported by the National Natural Science Foundation of China (11371230), SDUST Research Fund (2014TDJH102), Shandong Provincial Natural Science Foundation, China (ZR2015AQ001), Joint Innovative Center for Safe And Effective Mining Technology and Equipment of Coal Resources, SDUST Innovation Fund for Graduate Students (SDKDYC190351).

\section{References}

[1] Z. Liu and R. Tan, "Impulsive harvesting and stocking in a Monod-Haldane functional response predator-prey system," Chaos, Solitons \& Fractals, vol. 34, no. 2, pp. 454-464, 2007.

[2] D. V. Vayenas and S. Pavlou, "Chaotic dynamics of a food web in a chemostat," Mathematical Biosciences, vol. 162, no. 2, pp. 69-84, 1999.

[3] H. Liu and H. Cheng, "Dynamic analysis of a prey-predator model with state-dependent control strategy and square root response function," Advances in Difference Equations, vol. 2018, no. 1, p. 63, 2018.

[4] Z. Shi, Y. Li, and H. Cheng, "Dynamic analysis of a pest management smith model with impulsive state feedback control and continuous delay," Journal of Mathematical Chemistry, vol. 7, no. 7, p. 591, 2019.

[5] F. Wang and X. Zhang, "Adaptive finite time control of nonlinear systems under time-varying actuator failures," IEEE Transactions on Systems, Man, and Cybernetics: Systems, vol. 49, no. 9, pp. 1845-1852, 2019.

[6] J. Wang, H. Cheng, X. Meng, and B. S. A. Pradeep, "Geometrical analysis and control optimization of a predator-prey model with multi state-dependent impulse," Advances in Difference Equations, vol. 2017, no. 1, p. 252, 2017.

[7] M. Haque and E. Venturino, "The role of transmissible diseases in the Holling-Tanner predator-prey model," Theoretical Population Biology, vol. 70, no. 3, pp. 273-288, 2006.

[8] J. Yang and Y. Tan, "Effects of pesticide dose on Holling II predator-prey model with feedback control," Journal of Biological Dynamics, vol. 12, no. 1, pp. 527-550, 2018.

[9] Y. Huang, F. Chen, and L. Zhong, "Stability analysis of a preypredator model with Holling type III response function incorporating a prey refuge," Applied Mathematics and Computation, vol. 182, no. 1, pp. 672-683, 2006.

[10] Z. Shi, H. Cheng, Y. Liu, and Y. Li, "A cydia pomonella integrated management predator-prey model with smith growth and linear feedback control," IEEE Access, vol. 7, pp. 126066-126076, 2019.
[11] Z. Shi, H. Cheng, Y. Liu, and Y. Wang, "Optimization of an integrated feedback control for a pest management predatorprey model," Mathematical Biosciences and Engineering, vol. 16, no. 6, pp. 7963-7981, 2019.

[12] J. Jiao, X. Meng, and L. Chen, “A stage-structured holling mass defence predator-prey model with impulsive perturbations on predators," Applied Mathematics and Computation, vol. 189, no. 2, pp. 1448-1458, 2007.

[13] Y. Wang, H. Cheng, and Q. Li, "Dynamic analysis of wild and sterile mosquito release model with Poincaré map," Mathematical Biosciences and Engineering, vol. 16, no. 6, pp. 7688-7706, 2019.

[14] W. Lv and W. Fang, "Adaptive tracking control for a class of uncertain nonlinear systems with infinite number of actuator failures using neural networks," Advances in Difference Equations, vol. 2017, no. 1, p. 374, 2017.

[15] J. Sugie, "Two-parameter bifurcation in a predator-prey system of Ivlev type," Journal of Mathematical Analysis and Applications, vol. 217, no. 2, pp. 349-371, 1998.

[16] T. Ma, X. Meng, and Z. Chang, "Dynamics and optimal harvesting control for a stochastic one-predator-two-prey time delay system with jumps," Complexity, vol. 2019, Article ID 5342031, 19 pages, 2019.

[17] Y. Li, Y. Li, Y. Liu, and H. Cheng, "Stability analysis and control optimization of a prey-predator model with linear feedback control," Discrete Dynamics in Nature and Society, vol. 2018, Article ID 4945728, 12 pages, 2018.

[18] H. K. Baek, S. D. Kim, and P. Kim, "Permanence and stability of an Ivlev-type predator-prey system with impulsive control strategies," Mathematical and Computer Modelling, vol. 50, no. 9-10, pp. 1385-1393, 2009.

[19] F. Wang, Z. Liu, Y. Zhang, and C. L. P. Chen, "Adaptive finitetime control of stochastic nonlinear systems with actuator failures," Fuzzy Sets and Systems, vol. 374, pp. 170-183, 2019.

[20] J. Wang, H. Cheng, H. Liu, and Y. Wang, "Periodic solution and control optimization of a prey-predator model with two types of harvesting," Advances in Difference Equations, vol. 2018, no. 1, p. 41, 2018.

[21] Y. Li, H. Cheng, J. Wang, and Y. Wang, "Dynamic analysis of unilateral diffusion gompertz model with impulsive control strategy," Advances in Difference Equations, vol. 2018, no. 1, p. 32, 2018.

[22] Z. Shi, J. Wang, Q. Li, and H. Cheng, "Control optimization and homoclinic bifurcation of a prey-predator model with ratio-dependent," Advances in Difference Equations, vol. 2019, no. 1, p. 2, 2019.

[23] J. R. Beddington, "Mutual interference between parasites or predators and its effect on searching efficiency," The Journal of Animal Ecology, vol. 44, no. 1, pp. 331-340, 1975.

[24] D. L. Deangelis, R. A. Goldstein, and R. V. O’Neill, “A model for tropic interaction,” Ecology, vol. 56, no. 4, pp. 881-892, 1975.

[25] Y. Liu, H. Dong, and Y. Zhang, "Solutions of a discrete integrable hierarchy by straightening out of its continuous and discrete constrained flows," Analysis and Mathematical Physics, vol. 9, no. 1, pp. 465-481, 2019.

[26] T. Liu and H. Dong, "The prolongation structure of the modified nonlinear schrödinger equation and its initialboundary value problem on the half line via the RiemannHilbert approach,” Mathematics, vol. 7, no. 2, p. 170, 2019.

[27] H. Qi, L. Liu, and X. Meng, "Dynamics of a non-autonomous stochastic sis epidemic model with double epidemic hypothesis," Complexity, vol. 2017, Article ID 4861391, 14 pages, 2017. 
[28] Y. Li, H. Cheng, and Y. Wang, "A lycaon pictus impulsive state feedback control model with Allee effect and continuous time delay," Advances in Difference Equations, vol. 2018, no. 1, p. 367, 2018.

[29] J. Gu, Y. Zhang, and H. Dong, "Dynamic behaviors of interaction solutions of $(3+1)$-dimensional shallow water wave equation," Computers \& Mathematics with Applications, vol. 76, no. 6, pp. 1408-1419, 2018.

[30] X. Fan, Y. Song, and W. Zhao, "Modeling cell-to-cell spread of HIV-1 with nonlocal infections," Complexity, vol. 2018, Article ID 2139290, 10 pages, 2018.

[31] Z. Jiang, X. Bi, T. Zhang, B. S. A. Pradeep, and B. G. Sampath Aruna Pradeep, "Global hopf bifurcation of a delayed phytoplankton-zooplankton system considering toxin producing effect and delay dependent coefficient," Mathematical Biosciences and Engineering, vol. 16, no. 5, pp. 3807-3829, 2019.

[32] S. Liu, M. Huang, X. Song, S. Li, and H. Cheng, "Modeling impulsive resource inputs in host-parasitoid interactions with time delays," International Journal of Biomathematics, vol. 11, no. 5, Article ID 1850064, 2018.

[33] Y. Liu, X. Li, X. Feng, and L. Wang, "State-dependent pulse vaccination and therapeutic strategy in an SI epidemic model with nonlinear incidence rate," Computational and Mathematical Methods in Medicine, vol. 2019, Article ID 3859815, 10 pages, 2019.

[34] J. Wang, H. Cheng, Y. Li, and X. Zhang, "The geometrical analysis of a predator-prey model with multi-state dependent impulses," Journal of Applied Analysis and Computation, vol. 8, no. 2, pp. 427-442, 2018.

[35] J. Xu, Y. Tian, H. Guo, and X. Song, "Dynamical analysis of a pest management Leslie Gower model with ratio-dependent functional response," Nonlinear Dynamics, vol. 93, no. 2, pp. 1-16, 2018.

[36] Y. Tian, S. Tang, and R. A. Cheke, "Nonlinear state-dependent feedback control of a pest-natural enemy system," Nonlinear Dynamics, vol. 94, no. 3, pp. 2243-2263, 2018.

[37] W. Zhang, X. Meng, and Y. Dong, "Periodic solution and ergodic stationary distribution of stochastic siri epidemic systems with nonlinear perturbations," Journal of Systems Science and Complexity, vol. 32, pp. 1-21, 2019.

[38] T. Feng, Z. Qiu, and X. Meng, "Analysis of a stochastic recovery-relapse epidemic model with periodic parameters and media coverage," Journal of Applied Analysis and Computation, vol. 9, no. 3, pp. 1-15, 2019.

[39] T. Zhang, T. Zhang, and X. Meng, "Stability analysis of a chemostat model with maintenance energy," Applied Mathematics Letters, vol. 68, pp. 1-7, 2017.

[40] F. Liu, Z. Fu, and S. T. Jhang, "Boundedness and continuity of Marcinkiewicz integrals associated to homogeneous mappings on Triebel-Lizorkin spaces," Frontiers of Mathematics in China, vol. 14, no. 1, pp. 95-122, 2019.

[41] F. Wang, B. Chen, Y. Sun, Y. Gao, and C. Lin, "Finite-time fuzzy control of stochastic nonlinear systems," IEEE Transactions on Cybernetics, 2019.

[42] P. S. Simeonov and D. D. Bainov, "Orbital stability of periodic solutions of autonomous systems with impulse effect," International Journal of Systems Science, vol. 19, no. 12, pp. 2561-2585, 1988.

[43] M. Chi and W. Zhao, "Dynamical analysis of multi-nutrient and single microorganism chemostat model in a polluted environment," Advances in Difference Equations, vol. 2018, no. 1, p. 120, 2018.

[44] Y. Ren, M. Tao, H. Dong, and H. Yang, "Analytical research of $(3+1)$-dimensional rossby waves with dissipation effect in cylindrical coordinate based on lie symmetry approach," Advances in Difference Equations, vol. 2019, no. 1, p. 13, 2019.

[45] X. Meng, F. Li, and S. Gao, "Global analysis and numerical simulations of a novel stochastic eco-epidemiological model with time delay," Applied Mathematics and Computation, vol. 339, pp. 701-726, 2018.

[46] F. Liu, "Continuity and approximate differentiability of multisublinear fractional maximal functions," Mathematical Inequalities \& Applications, vol. 21, no. 1, pp. 25-40, 2018.

[47] T. Feng and Z. Qiu, "Global analysis of a stochastic TB model with vaccination and treatment," Discrete \& Continuous Dynamical Systems - B, vol. 24, no. 6, pp. 2923-2939, 2019.

[48] T.-W. Hwang, "Global analysis of the predator-prey system with Beddington-DeAngelis functional response," Journal of Mathematical Analysis and Applications, vol. 281, no. 1, pp. 395-401, 2003.

[49] T.-W. Hwang, "Uniqueness of limit cycles of the predatorprey system with Beddington-DeAngelis functional response," Journal of Mathematical Analysis and Applications, vol. 290, no. 1, pp. 113-122, 2004. 


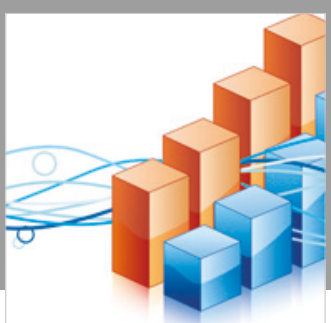

Advances in

Operations Research

\section{-n-m}
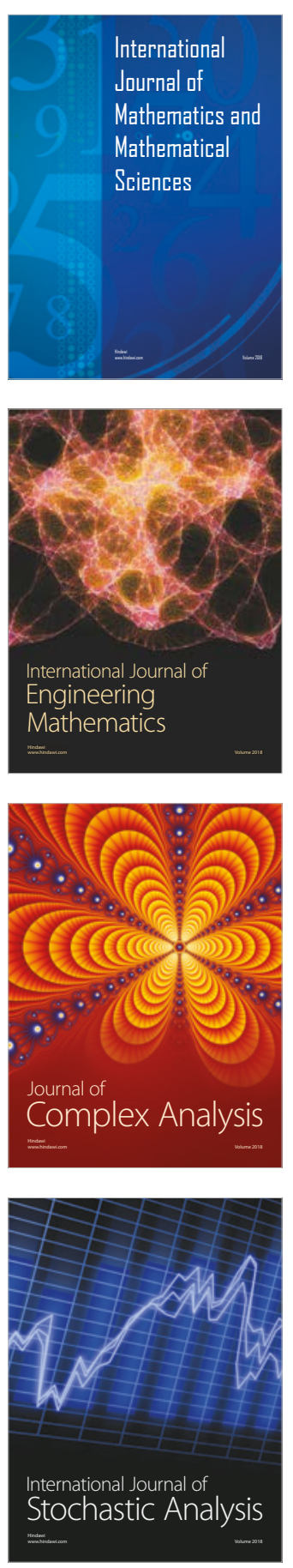
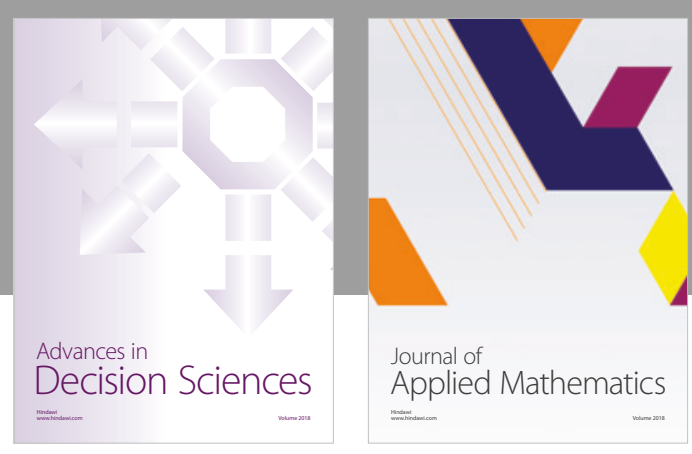

Journal of

Applied Mathematics
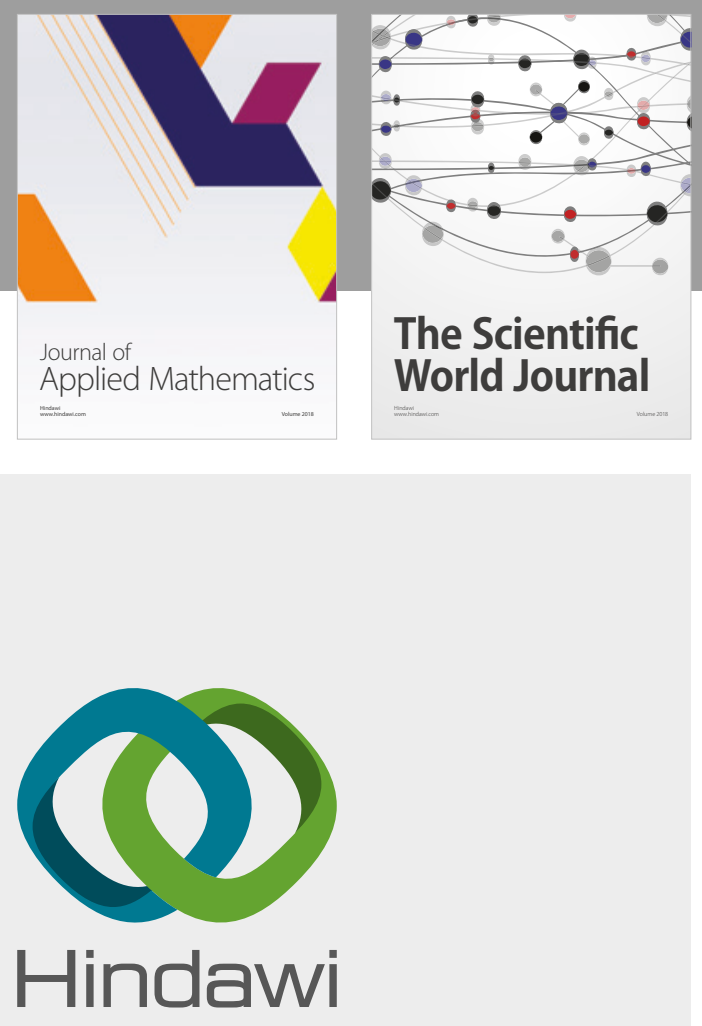

Submit your manuscripts at

www.hindawi.com

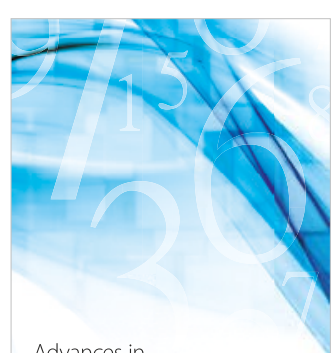

Advances in
Numerical Analysis
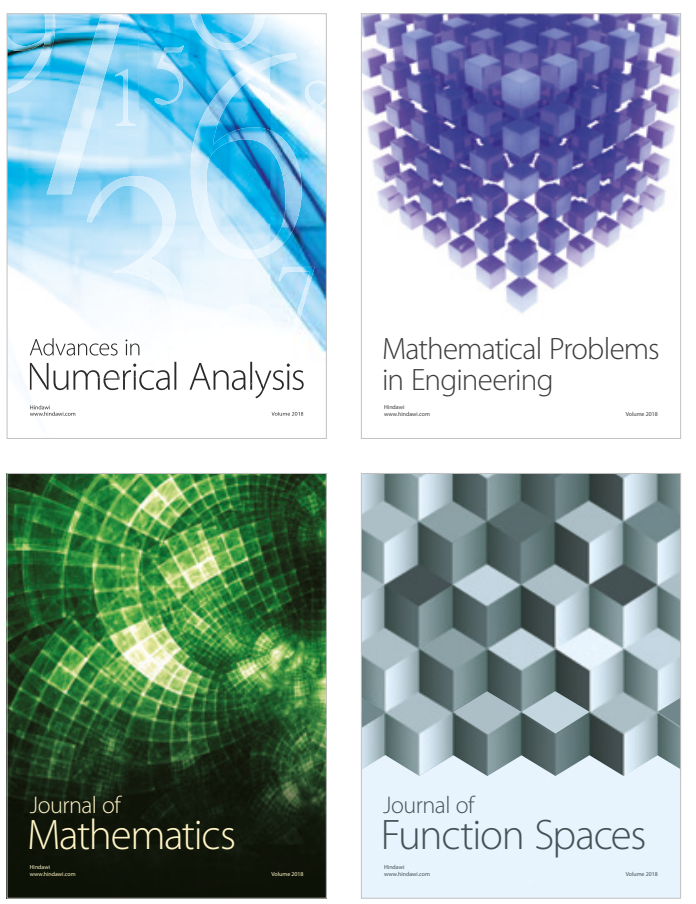

Mathematical Problems in Engineering

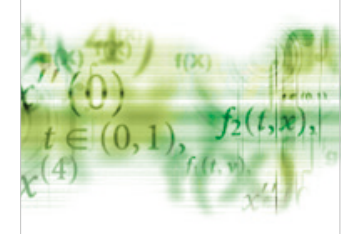

International Journal of

Differential Equations

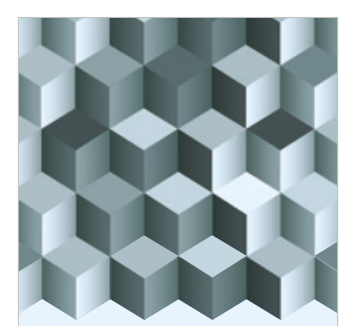

Journal of

Function Spaces
The Scientific

World Journal

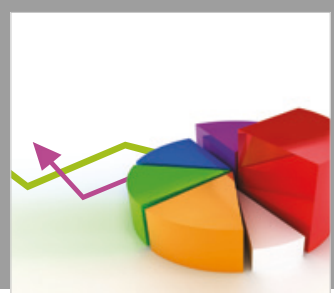

Journal of

Probability and Statistics
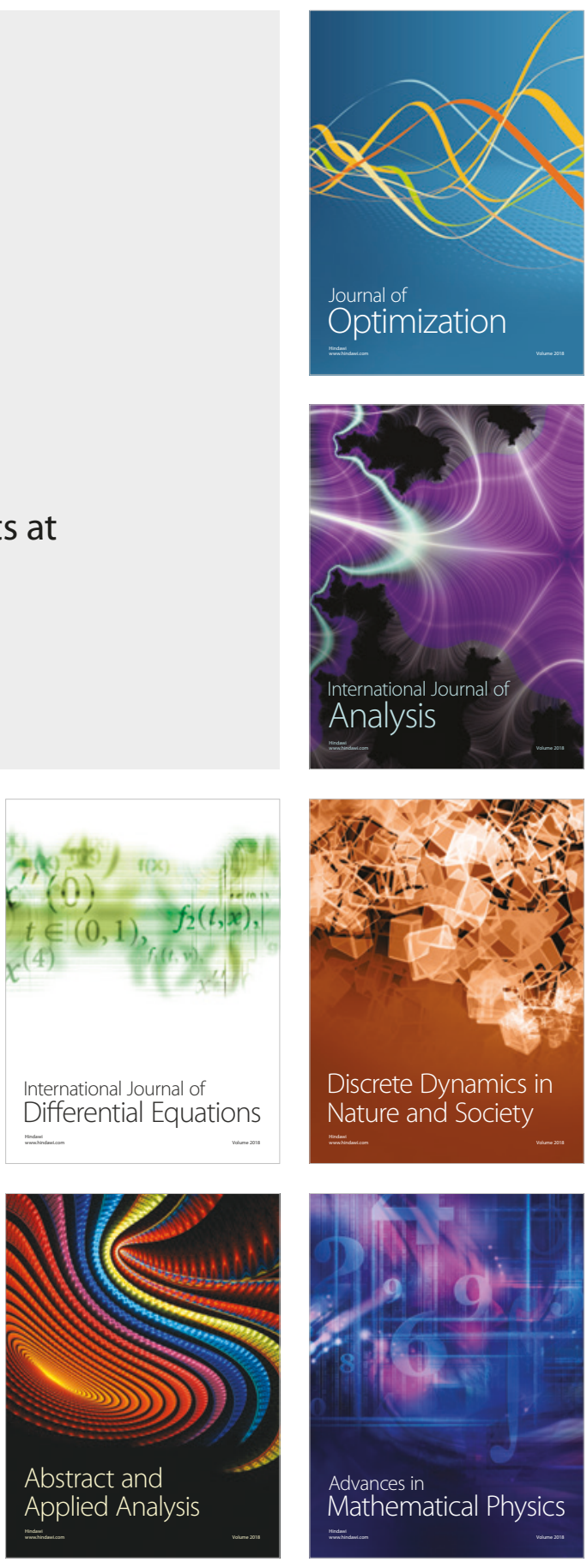